\title{
Neural Organization and Visual Processing in the Anterior Optic Tubercle of the Honeybee Brain
}

\author{
Theo Mota, ${ }^{1,2}$ Nobuhiro Yamagata, ${ }^{1,2}$ Martin Giurfa, ${ }^{1,2 *}$ Wulfila Gronenberg, ${ }^{3 *}$ and Jean-Christophe Sandoz ${ }^{1,2,4 \star}$ \\ Centre de Recherches sur la Cognition Animale, ${ }^{1}$ Université de Toulouse, Université Paul Sabatier and ${ }^{2}$ Centre National de la Recherche Scientifique \\ (CNRS), F-31062 Toulouse, France, ${ }^{3}$ Department of Neuroscience, University of Arizona, Tucson, Arizona 85721, and ${ }^{4}$ Laboratoire Evolution, Génomes et \\ Spéciation, CNRS, 91198 Gif-sur-Yvette, France
}

The honeybee Apis mellifera represents a valuable model for studying the neural segregation and integration of visual information. Vision in honeybees has been extensively studied at the behavioral level and, to a lesser degree, at the physiological level using intracellular electrophysiological recordings of single neurons. However, our knowledge of visual processing in honeybees is still limited by the lack of functional studies of visual processing at the circuit level. Here we contribute to filling this gap by providing a neuroanatomical and neurophysiological characterization at the circuit level of a practically unstudied visual area of the bee brain, the anterior optic tubercle $(\mathrm{AOTu})$. First, we analyzed the internal organization and neuronal connections of the AOTu. Second, we established a novel protocol for performing optophysiological recordings of visual circuit activity in the honeybee brain and studied the responses of AOTu interneurons during stimulation of distinct eye regions. Our neuroanatomical data show an intricate compartmentalization and connectivity of the $\mathrm{AOTu}$, revealing a dorsoventral segregation of the visual input to the AOTu. Light stimuli presented in different parts of the visual field (dorsal, lateral, or ventral) induce distinct patterns of activation in AOTu output interneurons, retaining to some extent the dorsoventral input segregation revealed by our neuroanatomical data. In particular, activity patterns evoked by dorsal and ventral eye stimulation are clearly segregated into distinct AOTu subunits. Our results therefore suggest an involvement of the AOTu in the processing of dorsoventrally segregated visual information in the honeybee brain.

\section{Introduction}

Processing visual information involves complex neurophysiological transformations achieved at successive stages within the brain. Many examples of such processing have been demonstrated in the visual system of insects, in which three visual neuropils-lamina, medulla, and lobula - provide consecutive stages of visual transformation and segregation before information is integrated in higherorder brain centers (fruit flies: Yamaguchi et al., 2008; calliphorid flies: Douglass and Strausfeld, 1998; locusts: Pfeiffer et al., 2005; bees: Paulk et al., 2008, 2009).

Honeybees represent an attractive model for studying the segregation and integration of different visual features in the insect brain. Visual cues are detected by compound eyes made of ommatidia, hosting nine photoreceptor cells each. Three types of

Received Feb. 23, 2011; revised June 1, 2011; accepted June 3, 2011.

Author contributions: M.G., W.G., and J.C.S. designed research; T.M. performed research; N.Y. contributed unpublished reagents/analytic tools; T.M., W.G., and J.C.S. analyzed data; T.M., M.G., W.G., and J.C.S. wrote the paper.

This work was funded by French National Research Agency Project 07-NEURO-003, APICOLOR (J.C.S.), National Science Foundation Grant IOB 0519483 (W.G.), the French Research Council (CNRS), and the University of Paul Sabatier. T.M. was supported by a doctoral scholarship from the CAPES Foundation and the Brazilian government. N.Y. was supported by the Fyssen Foundation. We are thankful to Patrick Arrufat for building the visual stimulation device, Maud Combe for programming the software for imaging analysis, and Edith Roussel for help with the analyses.

${ }^{*}$ M.G., W.G., and J.C.S. contributed equally to this work.

The author(s) declare(s) no competing financial interests.

Correspondence should be addressed to Jean-Christophe Sandoz, Laboratoire Evolution, Génomes, et Spéciation,

CNRS, 1 avenue de la Terrasse, 91198 Gif-sur-Yvette, France. E-mail: sandoz@legs.cnrs-gif.fr.

DOI:10.1523/JNEUROSCI.0995-11.2011

Copyright $\odot 2011$ the authors $\quad 0270-6474 / 11 / 3111443-14 \$ 15.00 / 0$ photoreceptors, S, M, and L (for short-, mid-, and long-range wavelength, respectively), peaking in the UV, blue, and green regions of the spectrum, respectively, have been identified in the honeybee retina (Peitsch et al., 1992; Wakakuwa et al., 2005). Most L photoreceptors terminate in the first neuropil of the optic lobe, the lamina, in which they synapse on lamina monopolar cells (Menzel, 1974). The lamina is composed of thousands of optical cartridges, each receiving an axon bundle that contains the axons of the nine photoreceptors from an overlying ommatidium, as well as the dendrites of different types of monopolar cells. Additionally, tangential, centrifugal, and horizontal fibers can be found within each cartridge. The spatial arrangement of photoreceptor axons and lamina monopolar cells within a cartridge remains constant throughout the lamina, thus providing the basis for a retinotopic organization. Axons of lamina monopolar cells and $\mathrm{M}$ and $\mathrm{S}$ receptors proceed to the second visual neuropil, the medulla, by way of the outer chiasm, which reverses retinotopic organization in an anteroposterior manner. Major output neurons from the medulla project via the inner chiasm to the third visual neuropil, the lobula. Extrinsic lobula neurons convey information to different brain regions, including the mushroom bodies (Mobbs, 1982, Ehmer and Gronenberg, 2002), the optic lobe of the contralateral eye (Hertel et al., 1987), and different subregions of the ipsilateral lateral protocerebrum (Hertel and Maronde, 1987).

Less is known about how visual information is processed in the lateral protocerebrum. Spherical neuropils termed optic glomeruli have been found in this region (flies: Strausfeld and 
Okamura, 2007; bees: Paulk et al., 2009), in which they are assumed to mediate visual processing (Strausfeld et al., 2007). The most prominent optic glomerulus is the anterior optic tubercle $(\mathrm{AOTu}) . \mathrm{AOTu}$ neurons involved in sky-compass, polarized light, and spectral processing have been characterized in the locust by means of intracellular recordings (Pfeiffer et al., 2005; Kinoshita et al., 2007). In honeybees, little is known about the neural architecture and function of the AOTu. Here we provide a neuroanatomical characterization of the honeybee AOTu and report the first neurophysiological measurements of AOTu responses at the network level by means of calcium imaging recordings performed during visual stimulation. We show that distinct subunits can be identified in the AOTu, which contribute to spatial vision by processing dorsoventrally segregated information.

\section{Materials and Methods}

Animals. Free-flying honeybee foragers (Apis mellifera, female workers) were caught at the entrance of an outdoor hive or directly from flowers situated close to the laboratory building. Bees were placed in small glass vials and cooled on ice until they ceased moving. For anatomical preparations with specific tracer injections (see below, Anatomy), bees were individually harnessed in small plastic tubes using low-temperature melting wax so that the head and the antennae could not move. For in vivo brain imaging, bees were fixed in plastic recording chambers (see below, Calcium imaging preparation). The head capsules were opened frontally, salivary glands and a small part of the tracheal sheath were removed, and the brain was exposed for dye-specific (anatomy) or calcium indicator-specific (calcium imaging) injections.

Anatomy. The general organization of the honeybee AOTu was studied from formaldehyde-fixed material stained with osmium tetroxide as described by Mares et al. (2005). Major pathways involving the AOTu were revealed by specific injections of fluorescent tracers (see below) directly into the AOTu or into distinct brain areas potentially connecting with the AOTu: the medulla, the lobula, the vertical lobes of the mushroom bodies, and the lateral accessory lobes (LALs) of the protocerebrum. Retrograde and anterograde staining was performed for observation of dendrites and axon terminals in each of the neuronal pathways. For tracer application, glass capillaries were pulled on an electrode puller (P-30; Sutter Instruments), and their tips were covered with a small amount of fluorescent tracer (see below) and then inserted into brain areas of interest (Ehmer and Gronenberg, 2002). For more specific injection in central brain regions, borosilicate thin-walled glass electrodes were backfilled with dye solution, inserted into the brain by help of a micromanipulator, and injected using a computer-controlled valve (Miniature Solenoid Valve; Lee Company). When the region of interest in the brain was reached, a small quantity of dye was injected. Dextrans (Invitrogen) labeled with Texas Red $(3000 \mathrm{kDa})$, tetramethyl-rhodamine $(10,000 \mathrm{kDa})$, fluorescein $(3000 \mathrm{kDa})$, Lucifer yellow $(10,000 \mathrm{kDa})$, or Alexa Fluor $(10,000 \mathrm{kDa})$ were used as tracers for single or doublestaining purposes. After tracer application, the small window opened in the head capsule was closed with the original piece of head cuticle and sealed with Eicosan (Aldrich). Animals were then fed with 50\% sucrose solution (wt/wt) and kept alive in a moist chamber for 12-24 h to allow tracer uptake and transport within the neurons. After diffusion, brains were dissected out and processed as described below.

Histology and image processing. Brains were fixed in phosphatebuffered paraformaldehyde solution (4\%), $\mathrm{pH} 6.8$, and dehydrated in ascending concentrations of ethanol or in dimethoxypropane (EMS). Specimens were then embedded in Spurr's low viscosity medium (EMS), sectioned at $15-20 \mu \mathrm{m}$ on a sliding microtome, and mounted on slides with Cytoseal (Apogent). Sections were viewed under bright-field or epifluorescence microscopy, respectively. Microscopic images were recorded at two to eight different focal depths (pseudo-optical sections) by using a SPOT 2 digital camera (Diagnostic Instruments). Pseudo-optical sections were merged into a single optical section using CombineZP (Hadley, 2010). Optical sections were merged and adjusted for brightness and contrast, and individual micrographs of adjacent brain areas were stitched together using Photoshop (Adobe Systems).
Part of the tracer-filled brains were cleared in methylsalicylate (SigmaAldrich) for $1 \mathrm{~d}$, and images of whole mounts were taken with a confocal laser scanning microscope (Leica SP5 with argon and DPSS 561 lasers) at $2 \mu \mathrm{m}$ optical section thickness. Projections of the confocal stacks, as well as double-staining overlay, were achieved with NIH ImageJ (Wayne Rasband, National Institutes of Health, Bethesda, MD). For 3D reconstructions, the entire AOTu surface was anteroposteriorly scanned at intervals of $2 \mu \mathrm{m}$ with $6 \times$ frame average. Complete stacks of optical sections $(512 \times 512$ pixels) were then imported into Amira 5.3 (Mercury Computer Systems). The AOTu compartments were reconstructed by manually tracing their contours on each section and then performing surface rendering. Brain areas and neuronal anatomy are described according to a neural axis in which the mushroom body calyces are dorsal, the antennal lobes ventral, and anterior, and the subesophageal ganglion posterior.

Calcium imaging preparation. For in vivo optophysiological recordings (Joerges et al., 1997; Sachse and Galizia, 2002; Deisig et al., 2010), bees were immobilized individually in an opaque plastic chamber (see Fig. $8 \mathrm{~A}$ ). For imaging the neural activity of inter-tubercle neurons, the calcium indicator fura-2 dextran (potassium salt, 10,000 kDa; Invitrogen) was introduced into the left AOTu (contralateral to visual stimulation). Three borosilicate thin-walled glass electrodes coated with crystals of the calcium indicator were consecutively inserted into the dorsal, medial, and ventral parts of the left AOTu. Fura-2 dextran migrated retrogradely to the right $\mathrm{AOTu}$ (ipsilateral to visual stimulation) within inter-tubercle neurons. This allowed measuring changes in dendritic calcium concentration of these neurons in the right AOTu corresponding to the stimulated right eye of the bee.

After fura-2 dextran injections, the head capsule was closed, and animals were fed with $50 \%$ sucrose solution and kept alive in a moist chamber for $18 \mathrm{~h}$ to allow calcium-indicator uptake and retrograde transport to the AOTu on the recording side. Small pieces of black plastic foil were waxed to the head (see Fig. $8 \mathrm{~A}$ ), and small remaining gaps were closed with epoxy glue (Red Araldite; Bostik Findley) mixed with an organic black pigment (Noir de Vigne; Frère Nordin). These plastic barriers created a small pool around the brain region, which could be kept under saline at all times, and also optically isolated the compound eye on the stimulated side. The opaque plastic barriers ensured that visual stimuli did not interfere with the imaging CCD camera and, reversely, that the microscope imaging light did not stimulate the eyes. The eye on the injection side (contralateral to visual stimulations) was covered with black epoxy. Finally, the small window in the head capsule was reopened, and the brain was immersed in saline solution [in mmol/l: $130 \mathrm{NaCl}, 6$ $\mathrm{KCl}, 4 \mathrm{MgCl}_{2}, 5 \mathrm{CaCl}_{2}, 160$ sucrose, 25 glucose, and 10 HEPES, pH 6.7 (500 mOsm) (all chemicals from Sigma-Aldrich)].

Visual stimulation. Light stimulation (see Fig. $8 B$ ) was produced by three rectangular arrays of 15 white light-emitting diodes (LEDs) (CREE LED Light) presented to different parts of the visual field of the bees' right eye (recording side). The LEDs (400-650 nm emission, with two peak wavelengths at 439 and $556 \mathrm{~nm}$ ) were suited to stimulate essentially the $\mathrm{M}$ (blue) and L (green) photoreceptors but not the S (UV) photoreceptors, whose spectral sensitivity precludes significant excitation at the photon flux used for stimulation (see below). The three LED arrays $(2.5 \times 1.8 \mathrm{~cm}$ each, in the vertical and horizontal directions, respectively) were arranged in a semicircle around the stimulated eye so that the central axes of the arrays pointed to the dorsal, lateral, or ventral part of the visual field, respectively (see Fig. $8 \mathrm{~B}$ ). The center of each array was placed at $6 \mathrm{~cm}$ from the stimulated eye so that each array had an angular subtense at the bee eye of $24.5^{\circ}$ and $17.1^{\circ}$ in the vertical and the horizontal directions, respectively (see visual angle calculation by Giurfa et al., 1996). The intensities of all three white LED arrays were adjusted with individual resistors so that each array had an irradiance of $7.61 \times 10^{6}$ photon counts $/ \mathrm{cm}^{2} / \mathrm{s}$ at the level of the bee's eye, measured using a fixed grating spectrometer (Ocean Optics S2000) with a DT1000 mini light source (200-1100 nm) and R400-7 UV/VIS optical probe (Ocean Optics). Because the sensitivity of the spectrometer (in the range of 400-650 $\mathrm{nm}$ ) is estimated as $\sim 86$ photons/count $/ \mathrm{ms}$, each array had an irradiance of $\sim 6.55 \times 10^{8}$ photons $/ \mathrm{cm}^{2} / \mathrm{s}$. Each light stimulus (dorsal, lateral, or ventral array) was presented for $2 \mathrm{~s}$ during imaging recordings (see below). Control stimuli consisted of identical recordings without any light 
presentation. The order of visual stimulus presentation and control recordings was randomized within and between bees. Recordings with each visual stimulus as well as control recordings were replicated three times per bee. The minimum interstimulus interval was $1 \mathrm{~min}$, thus avoiding potential adaptation to light stimuli (Paulk et al., 2008).

Recordings of visual-evoked activity. Calcium imaging recordings were performed in standard conditions (Sachse and Galizia, 2002; Deisig et al., 2010) using a T.I.L.L. Photonics imaging system. Bees previously injected with fura-2 dextran were placed under an epifluorescent microscope with a $20 \times$ water-immersion objective (NA 0.5) immersed in the saline solution covering the brain. Brain movements were reduced by squeezing the bee's abdomen with soft modeling clay. The AOTu on the stimulation side was imaged. Images were taken using a $640 \times 480$ pixel 12-bit monochrome CCD camera (T.I.L.L. Imago) cooled to $-12^{\circ} \mathrm{C}$. Each measurement consisted of 60 frames at a rate of $5 \mathrm{~Hz}$ (interval between frames, $200 \mathrm{~ms}$ ). Imaging excitation light was shut off between frames. Visual stimulation was given at the 15 th frame for $2 \mathrm{~s}$. Pixel image size corresponded to $2.4 \times 2.4 \mu \mathrm{m}$ after $4 \times 4$ binning on chip. Monochromatic excitation light alternating between 340 and $380 \mathrm{~nm}$ was applied using a monochromator (T.I.L.L Polychrom IV). The filter set on the microscope was composed of a $490 \mathrm{~nm}$ dichroic filter and a bandpass $(50 \mathrm{~nm}) 525 \mathrm{~nm}$ emission filter. The integration time was $6-40 \mathrm{~ms}$ for $380 \mathrm{~nm}$ stimulation and $18-160$ for $340 \mathrm{~nm}$ stimulation.

Analysis of activity signals and signal amplitudes. Calcium imaging data were analyzed using custom-made software written in IDL 6.4 (Research Systems) and following standard methods (Galizia and Vetter, 2005; Deisig et al., 2006, 2010) unless stated otherwise. Fura-2 is a ratiometric dye, and therefore each recording was a four-dimensional array with the excitation wavelength (340 and $380 \mathrm{~nm})$, two spatial dimensions $(x, y$ pixels of the area of interest), and the temporal dimension (60 frames). For calcium-signal analysis, each visual stimulation recording was brought to a three-dimensional array by calculating the fluorescence ratio $R=F_{340 \mathrm{~nm}} / F_{380 \mathrm{~nm}}$ at each pixel and time point. Then, three steps were performed to calculate the signals. First, relative fluorescence changes were calculated as $\Delta R / R=\left(R-R_{0}\right) / R_{0}$, taking as reference background $R_{0}$ the average of five frames just before any visual stimulation (here, frames 10-14). Second, to reduce photon noise, the raw data were filtered in the temporal and the two spatial dimensions using a median filter with a size of three pixels. Third, we applied a bleach correction. We subtracted a logarithmic curve fitted to the median brightness decay of the entire image frames, excluding the frames during the stimulus until $5 \mathrm{~s}$ after stimulus onset. Signal amplitude was calculated between the baseline just before the stimulus (average of frames 12-14) and $1 \mathrm{~s}$ after stimulus onset, when signal is maximum (average of frames 19-21). For display, activity signals were low-pass filtered (median, $9 \times$ 9) and presented in a false-color code, from dark blue (no signal) to red (maximum signal). For quantitative analysis of the data, signal amplitude at particular points of interest was calculated by averaging $9 \times 9$ pixels, which corresponded in both cases to a $\sim 20 \times 20 \mu \mathrm{m}$ region.

Stimulus-specific activity in the AOTu. To determine which pixels were statistically activated by each visual stimulus, we used a threshold-based method. First, signals were cropped to a region close to the borders of the $\mathrm{AOTu}$, and the ratio $R=F_{340 \mathrm{~nm}} / F_{380 \mathrm{~nm}}$ at each pixel and time point was calculated. Afterward, $\Delta R / R=\left(R-R_{0}\right) / R_{0}$ was computed, and bleaching was corrected as above. Then, data were low-pass filtered (Gaussian $13 \times 13)$. To analyze the activated pixels in specific subregions of the AOTu [dorsal lobe (MU-DL) and ventral lobe (MU-VL) of the major units], signals were cropped to the region of interest, and calculation of $\Delta R / R$ was performed as above.

A pixel was considered to be activated if it fulfilled two criteria: (1) activation amplitude was above noise level and (2) the pixel was part of a group of at least five activated pixels to ensure that it revealed coherent neural activity. For each bee, noise was determined as a number of SDs of the signal before (i.e., frames 1-14) the stimulus. Because of differences between bees in the overall quality of the recordings, a noise threshold was determined for each bee (between 2 and 4 SDs) depending on the overall signal amplitude level. The same threshold was applied to all recordings of the same bee. Using the treatment described here, clear patterns of visually evoked activity could be revealed in the AOTu structure.

Overlap between stimulus-specific AOTu activity maps. To test the existence of stimulus-specific activity and coding in the AOTu, we compared the overlap of activated pixels between stimulations in the same or in different parts of the visual field (dorsal, lateral, or ventral). Overlap between two visually evoked recordings was calculated as the number of activated pixels common to the two recordings (intersection) divided by the number of pixels activated in both recordings (union). In other words, for two recordings $\mathrm{A}$ and $\mathrm{B}$, Overlap $=\cap_{\mathrm{A}, \mathrm{B}} / \mathrm{U}_{\mathrm{A}, \mathrm{B}}$. We calculated for each bee the average overlap between any two recordings of light stimulations in the same part of the visual field $\left(\right.$ Overlap $\left._{\text {same }}\right)$ or in two different parts of the visual field (Overlap different $_{\text {t }}$ ).

Statistical analysis. Friedman's tests followed by Wilcoxon's matchedpairs tests were used for comparing the following: (1) amplitudes of calcium signals obtained in the AOTu to stimulations of different parts of the visual field and control stimuli (see Fig. 8E), (2) overlap measures obtained from different pairs of stimulations (see Fig. 9D), and (3) numbers of activated pixels in each AOTu subcompartment to different stimulations (see Fig. 9E). Pairwise comparisons were performed using a Bonferroni's correction for multiple comparisons. The Overlap same and Overlap $_{\text {different }}$ measures calculated over all bees were directly compared using a Wilcoxon's matched-pairs test (see Fig. 9B).

\section{Results}

We have characterized the anatomical organization of the AOTu in the honeybee and performed the first physiological measurements of neural activity at the network level in this structure. For the latter, we used a novel optical imaging approach that allowed recording response characteristics of AOTu output neurons during visual stimulation. We first present our anatomical findings and then focus on physiological responses.

\section{AOTu location and compartmentalization}

The AOTu resides close to the anterior brain surface, dorsal to the antennal lobe and ventrolateral to the vertical lobe of the mushroom bodies. Figure $1 A$ represents the anteriormost 50 $\mu \mathrm{m}$ of the brain and shows the superficial position of the AOTu (boxes). The bulk of the AOTu is formed by a unit that appears almost circular in successive frontal cross-sections as shown in Figure $1 A-E$. This part is here referred to as MU, the major unit. Vertical sections (from anterior to posterior) (Fig. $1 F, G)$ show that the MU is not spherical but slightly flattened in the anteroposterior axis. It can be observed from the most anterior brain sections (Fig. $1 C$ ) up to a depth of $\sim 80 \mu \mathrm{m}$ (Fig. $1 D, E)$ in the protocerebrum.

An enlargement of the osmium-stained frontal sections (Fig. $1 B)$ reveals a compartmentalization of the MU. In its anterior part, the MU is symmetrically subdivided in MU-VL and MU-DL by a narrow band that appears less intensely stained in osmium preparations (Fig. $1 B, C$ ). Toward the posterior end of the MU, the two subcompartments (MU-VL and MU-DL) are fused (Fig. $1 D, E)$ so that VL and DL are not completely separated.

Two smaller units are situated posterior to the MU: a ventrolateral unit (VLU) (depth, 50-95 $\mu \mathrm{m}$ from the anterior brain surface) and a lateral unit (LU) (depth, $\sim 55-105 \mu \mathrm{m}$ ). The small VLU (Fig. $1 B, D, E$ ) resides on the posterior lateral side of the MU-VL. The elongated lateral unit (Fig. $1 D, E)$ is located on the lateral side of the MU, at the level of the central band separating MU-VL and MU-DL. Its full extent can be best observed in vertical sections (Fig. $1 G$ ). Figure $2 \mathrm{~A}$ shows a three-dimensional reconstruction of the AOTu with its different subunits based on confocal sections of whole-brain mounts. 


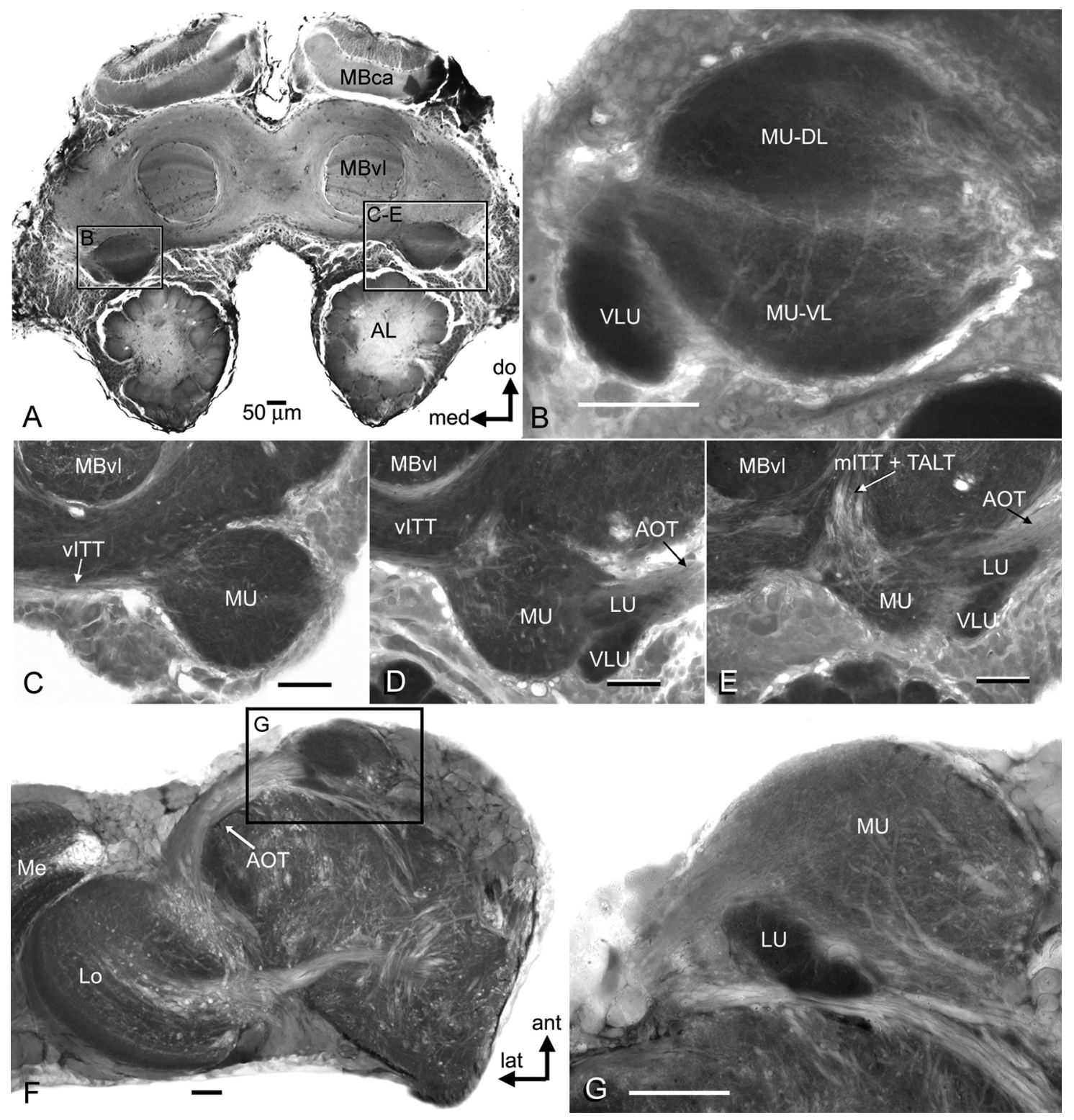

Figure 1. Position and internal organization of the AOTu in osmium-stained sections. $A, 0$ verview (frontal section) of the anteriormost part of the bee brain showing the position of the right ( $B$ ) and left $(\boldsymbol{C}-\boldsymbol{E})$ AOTus. Boxed areas enlarged in $\boldsymbol{B}$ and $\boldsymbol{C}-\boldsymbol{E}$, respectively. $\boldsymbol{B}$, Enlargement revealing the internal compartmentalization of the right A0Tu. $\boldsymbol{C}-\boldsymbol{E}$, Frontal sections of the left A0Tu at different depths from the anterior brain surface $(\boldsymbol{C}, 30 \mu \mathrm{m} ; \boldsymbol{D}, 60 \mu \mathrm{m} ; \boldsymbol{E}, 80 \mu \mathrm{m}) . \boldsymbol{F}$, Vertical section of the left brain hemisphere; boxed area enlarged in $\boldsymbol{G}$. $\boldsymbol{G}$, Detail of the A0Tu vertically sectioned through the center of the MU and revealing the posteriorly positioned LU. AL, Antennal lobe; MBvl, mushroom-body vertical lobe; MBca, mushroom-body calyx; Me, medulla; Lo, lobula; med, medial; do, dorsal; ant, anterior; lat, lateral. Scale bars, $50 \mu \mathrm{m}$.

\section{Pathways connecting the AOTu with other brain regions}

General neuronal staining and specific dextran-coupled dye injections allowed identifying four distinct tracts connecting the AOTu with other brain areas (Fig. $2 B$ ). The prominent anterior optic tract (AOT) (Figs. $1 D-F, 2 B$ ) conveys information from the medulla and the lobula. Furthermore, two distinct tracts interconnect the AOTus of both brain hemispheres: the ventral inter-tubercle tract (vITT) (Figs. 1C,D,2B) and the medial intertubercle tract (mITT) (Figs. 1E, 2B). Last, the tubercle-accessory lobe tract (TALT) (Figs. 1E, 2B) connects the AOTu with the lateral accessory lobe in the median protocerebrum. In addition to these four tracts, we identified a specific neuron previously described as the A5-2 neuron (Rybak and Menzel, 1993), which connects the vertical lobe of the mushroom bodies with the AOTu (Fig. 2 B). Each of these distinct neuronal pathways, their projections in the brain, and the specific features of their neurons were studied in detail using localized dextran-coupled tracer injections.

\section{The AOT provides segregated input from the optic lobe to the AOTu}

The AOTu receives visual input from the medulla and lobula via the AOT (Figs. $1 F, 2 B$ ), which projects ventroanteriorly from the proximal lobula toward the AOTu. Tracer injections in the AOTu (retrograde labeling) revealed that the AOT comprises a large, mixed population of transmedullary neurons and lobula neurons (Fig. 3). All these retinotopic (columnar) small-field neurons converge to form the massive AOT arising from the inner edge of the lobula (Fig. $1 F, 3 A, D, H$ ).

The cell bodies of transmedullary neurons are situated along the distal face of the medulla (Figs. $3 A, 4 A$ ), from where the neurites proceed proximally. On reaching the serpentine layer of 

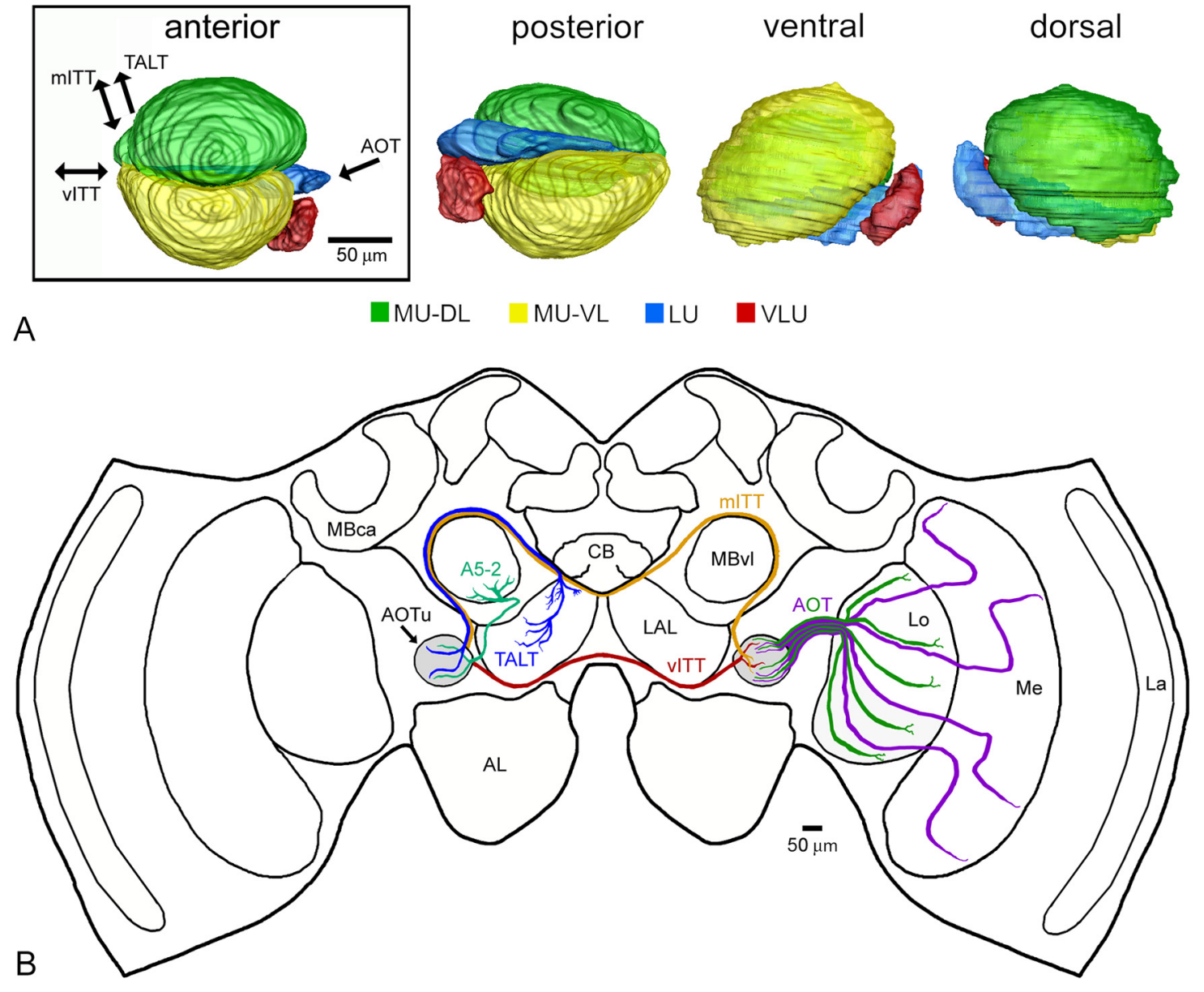

Figure 2. Three-dimensional structure and neural connectivity of the A0Tu. $A$, Three-dimensional reconstruction of confocal image stacks (anterior, posterior, ventral, and dorsal views) showing the different AOTu compartments: MU-DL, green; MU-VL, yellow; VLU, red; LU, blue. This example shows a left AOTu. $B$, Schematic diagram summarizing neural pathways connecting the AOTu with other brain neuropils. La, Lamina; Me, medulla; Lo, lobulla; AL, antennal lobe; MBvl, mushroom-body vertical lobe; MBca, mushroom-body calyx; CB, central body; LAL, lateral accessory lobe.

the medulla, which is made exclusively of fibers, many neurites run a certain distance within it and then exit it again, thus forming two right angle bends (Fig. $3 B, C$ ). These transmedullary neurons appear to have very short and inconspicuous dendrites that probably contact a single or only a few columns in the medulla layers distal to the serpentine layer (Fig. $3 B, C$; because of their small size, the short dendritic branches can barely be resolved in C). Transmedullary neurons run toward the lobula and are bundled in many fascicles at the level of the inner chiasm between medulla and lobula (Figs. 3A, 4A). We also typically stained a number of fibers originating from the dorsalmost part of the medulla and entering the dorsalmost part of the lobula trough the inner chiasm (Figs. 3A, 4A, red arrow). These neurons probably receive information from the long visual fibers of the photoreceptors in the dorsal rim area of the compound eye, whose axons terminate in the dorsalmost part of the medulla and most of which respond to polarized ultraviolet light (Labhart, 1980) (for review, see Labhart and Meyer, 2002).

Simultaneous injections of two tracers with different colors, one into the dorsal and the other into the ventral medulla, showed that AOT projections from the medulla are segregated in the AOTu (Fig. $3 D, E$ ). Neurons from the ventral medulla only project to the MU-VL and to the dorsal part of the MU-DL (Fig. $3 D, E$ ) (schematized in Fig. 10). Conversely, neurons from the dorsal medulla only project to the ventral part of the MU-DL and to the VLU (Fig. 3D,E) (schematized in Fig. 10).
Retrograde fills from the AOTu also labeled a large number of neurons (Fig. $3 A, F, G$ ) branching in all the distinct lobula layers (Ribi and Scheel, 1981). Although our mass fills did not allow us to precisely follow individual neurons, we were able to observe some dendrites of lobula tangential neurons in proximal layers 5 and 6 (Fig. $3 A, F$ ), as well as numerous dendrites of small-field (columnar) neurons in distal layers $1-4$ (Fig. $3 A, F, G$ ). Similarly to the medulla input, a dorsoventral segregation of the lobula input to the AOTu was identified when dextran-coupled tracer injections were simultaneously performed in the ventral and dorsal lobula, respectively (Fig. $3 H, I$ ). Neurons from the ventral lobula project mainly to the dorsal part of the MU-DL but also to the dorsal part of the MU-VL (Fig. $3 H, I$ ) (schematized in Fig. 10). Conversely, neurons from the dorsal lobula project to the ventral part of the MU-DL and to the MU-VL (Fig. 3 H,I) (schematized in Fig. 10). Although the dorsoventral segregation of the lobula input is very distinct in the MU-DL, the segregation of fibers from the dorsal and ventral lobula is less pronounced in the dorsal part of the MU-VL (Fig. 3 H,I). Because there are so many columnar neurons in the lobula, we were not able to follow the (fewer) wide-field neurons of the proximal lobula. Therefore, we do not know where these wide-field neurons terminate within the AOTu.

These anatomical findings demonstrate that the AOTu receives a large amount of small-field input from the medulla and 
lobula via the AOT and support the idea that information originating from dorsal and ventral eye regions remains primarily segregated at the level of the AOTu input. Our tracer studies did not allow us to discriminate the dorsoventral input segregation from a more fine-grained, retinotopic representation of optic lobe input to the AOTu.

\section{The vITT provides interhemispheric} connection between both AOTus

The AOTus on either side of the brain are interconnected by the vITT (Figs. $1 C, D$, $2 B, 4 A, B)$, a prominent tract that projects ventrally and superficially close to the esophageal foramen. Tracer injections into one of the two AOTus indicate that the vITT comprises $\sim 27$ axons (each with input in one AOTu and output in the other). The axons of vITT neurons are thicker than the ones of AOTu input neurons from the optic lobe. Neurons connecting the two AOTus enter the AOTu at the inner, anterior side of the MU and bifurcate, sending distinct arborizations in the ventral and dorsal lobes of the MU (Fig. $4 D, E$ ). Because of their symmetric arrangement in the two AOTus, we were not able to discriminate dendritic and axonal terminals as both kinds were always stained by injecting into the contralateral AOTu. We can therefore speculate that $\sim 13-14$ of these neurons exist per AOTu.

In many of our preparations, we found a particular pair of large interlobulae neurons running within or nearby the AOT of both brain hemispheres and in close proximity to the vITT (Fig. 4F). These two anterior interlobulae neurons do not seem to have projections anywhere else in the brain except for the ipsilateral (input) and contralateral (output) lobula (DeVoe et al., 1982; Hertel et al., 1987). Hence, these neurons are not associated or connected with the AOTu.

\section{The mITT provides additional} interhemispheric connection between both AOTus

A second tract, the mITT (Figs. $1 E, 2 B$, $4 A-C$ ) runs much more posteriorly in the lateral protocerebrum and projects dorsally through the protocerebrum and around the vertical lobes of the ipsilateral and contralateral mushroom bodies before projecting ventrally again to terminate in the contralateral AOTu (Fig. 4B,C). Centrally, between the two vertical mushroom body lobes, the mITT does not run straight but sags a bit ventrally (Fig. 4B,C). Despite the difficulty to follow individual neurons in mass-fill preparations of the mITT, we could identify a number of small and very thin lateral branches at the level of the median protocerebrum, suggesting additional connection with this brain region (Fig. $4 C$ ). The mITT comprises fewer but
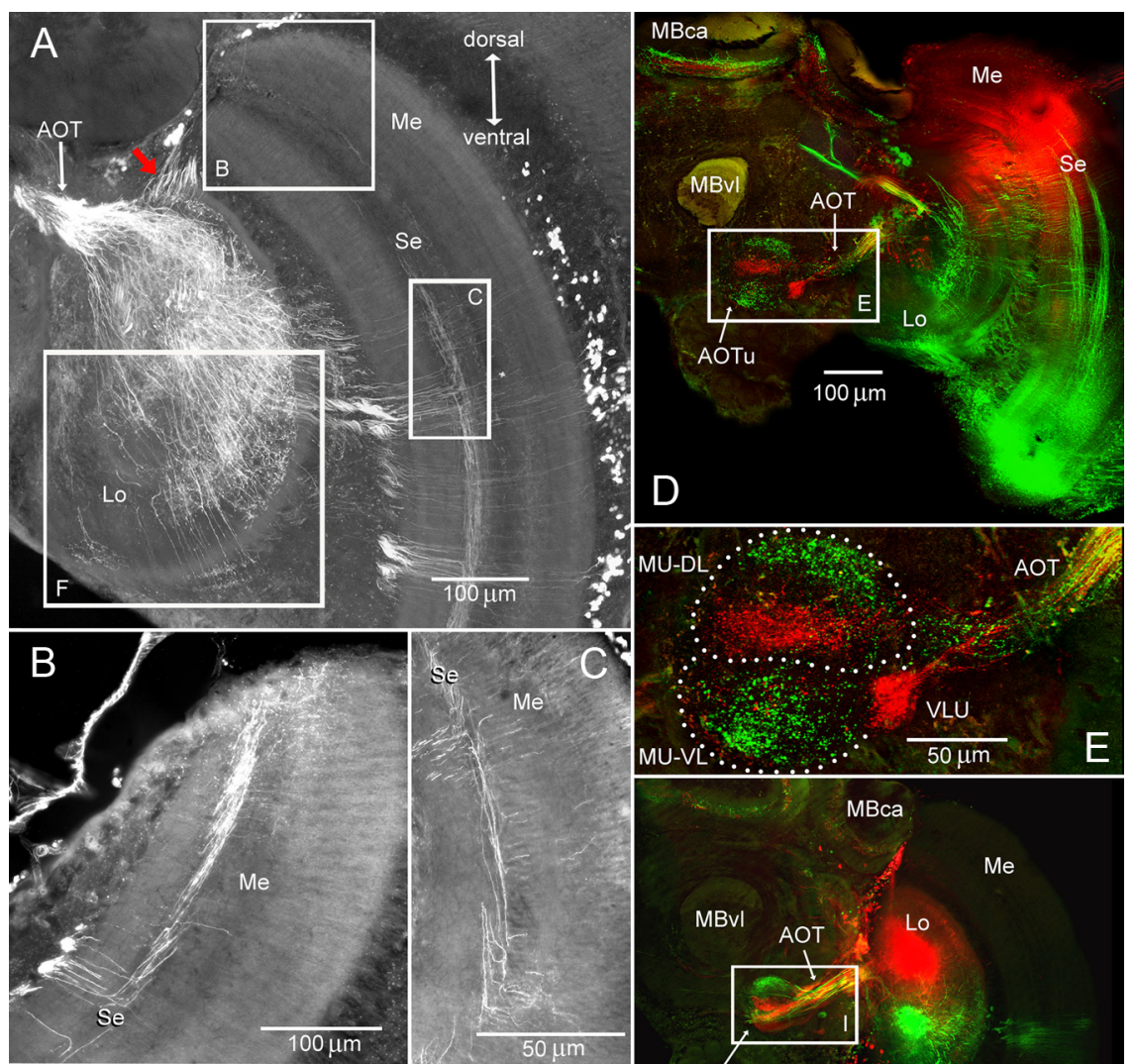

Figure 3. Segregated input from the optic lobe to the AOTu. $\boldsymbol{A}$, Dextran tracer injection into the A0Tu (as in Fig. $4 \mathrm{~A}$ ) reveals visual input from the medulla (Me) and lobula (Lo) via the AOT. Transmedullary neurons have cell bodies situated along the distal face of the medulla, traverse the medulla from distal to proximal featuring putative input regions in the outer medulla and usually short tangential diversions from their centripetal course within the serpentine (Se) layer $(\boldsymbol{B}, \boldsymbol{C})$, and are bundled in many fascicles on reaching the inner chiasm. Red arrow in $\boldsymbol{A}$ indicates fibers originating from the dorsalmost part of the medulla and entering the dorsalmost part of the lobula; boxed areas represent comparable regions from different preparations detailed in $\boldsymbol{B}, \boldsymbol{C}$, and $\boldsymbol{F}$, respectively. $\boldsymbol{D}$, Simultaneous injections of two tracers with different colors into the dorsal (red) and ventral (green) medulla show that projections from the medulla are segregated into different AOTu regions; boxed area enlarged in $\boldsymbol{E}$. Lobula neurons sending input to the AOTu $(\boldsymbol{A})$ are mainly columnar neurons with dendrites in proximal layers $1-4(\boldsymbol{F}, \boldsymbol{G})$. $\boldsymbol{H}$, Dorsoventral segregation of input to the AOTu was also identified when tracer injections were simultaneously performed in the ventral (in red) and dorsal (in green) lobula. Boxed area enlarged in $I$; abbreviations are as defined in Figures 1 and 2.

thicker axons compared with the vITT (Fig. $4 A-C$ ). Counts of fiber profiles at the location where the mITT enters the contralateral AOTu revealed six fibers. Because the same number of axons runs in the opposite direction, the mITT comprises at least 12 major axons, which show several thick arborizations as they enter the AOTu (Fig. 4C,E).

Arborizations of inter-tubercle neurons (Fig. 4D,E) are clearly segregated in the different AOTu compartments. Whereas the vITT enters the major unit of the AOTu anteriorly (Fig. $4 D, E)$, the mITT enters the AOTu dorsolaterally at the inner, more posterior face of the MU (Fig. $4 E$ ). Staining of all ITT 

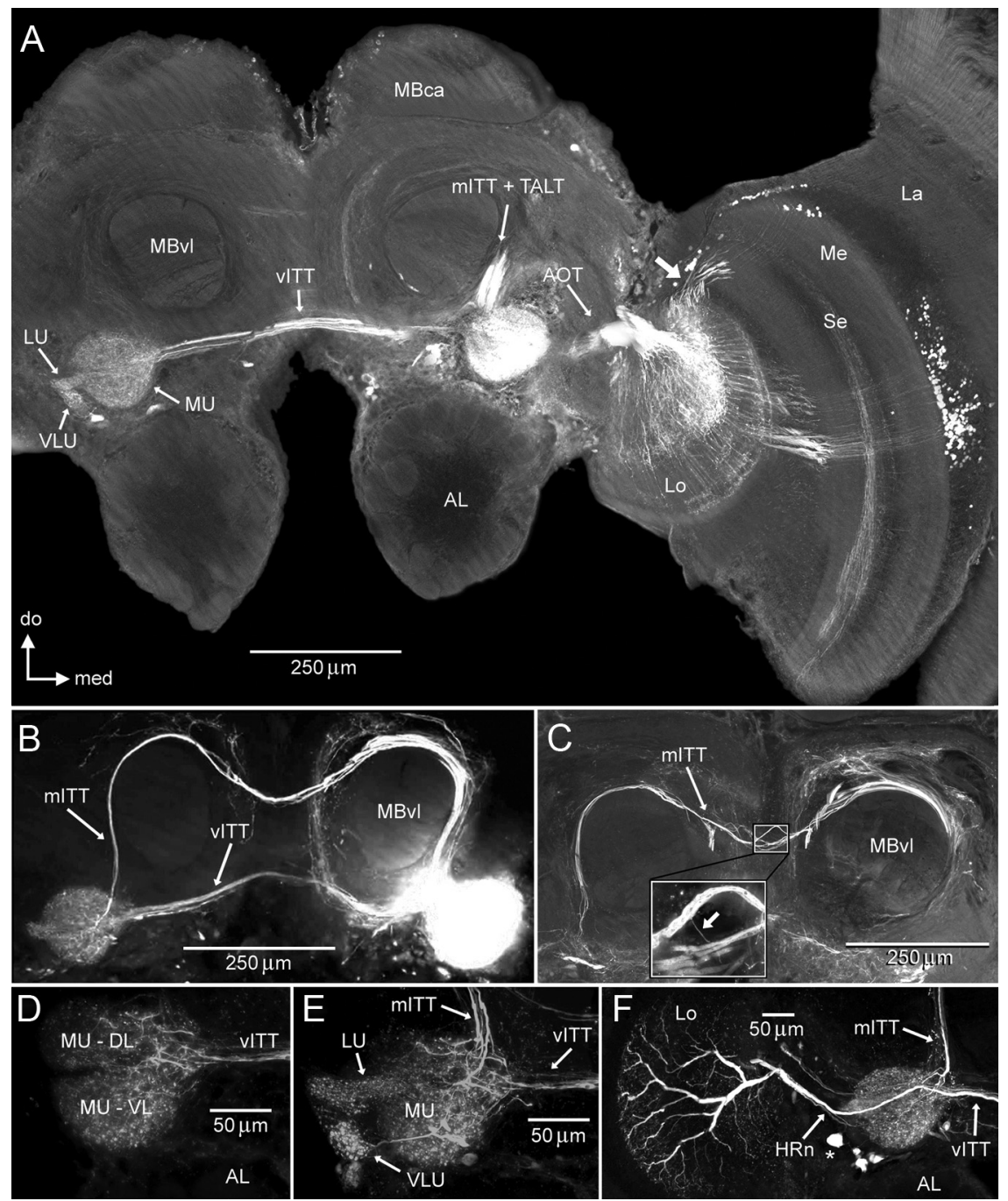

Figure 4. Two separate inter-tubercle tracts connecting the AOTs of both brain hemispheres. $A$, Composite of frontal sections after tracer injection into the AOTu showing visual input from the optic lobe (see details in Fig. 3) and inter-tubercle connection. The thick arrow indicates input fibers originating from the dorsalmost part of the medulla. The vITT runs ventrally in the anterior surface of the brain, whereas the mITT runs laterally together with the TALT. $B$, Superposition of frontal protocerebral sections showing vITT and mITT stained after tracer injection into the left AOTu. The mITT is posterior in relation to the vITT and projects dorsally through the protocerebrum, making a loop around the vertical lobes of the ipsilateral and contralateral mushroom bodies (MBvl) before projecting ventrally again to terminate in the contralateral $\mathrm{AOTu}$. $C$, $\mathrm{mITT}$ neurons possess very thin collaterals in the central brain (thick arrow in inset). $\boldsymbol{D}, \boldsymbol{E}$, Arborizations of inter-tubercle neurons in distinct AOTu compartments. Frontal section of the AOTu anterior $(\boldsymbol{D})$ and posterior $(\boldsymbol{E})$ side after tracer injection in the contralateral AOTu. All four AOTu compartments are filled with arborizations of inter-tubercle neurons. Whereas the vITT enters the MU anteriorly, the mITT enters the AOTu dorsolaterally at the inner, more posterior face of the MU. $\boldsymbol{F}$, An interlobulae neuron previously described as horizontal regressive motion sensitive (HR-n) by DeVoe et al. (1982) runs in very close proximity to the vITT trajectory. The large HR-n bypasses the posterior medial side of the AOTu and possesses a large cell body (asterisk) situated ventrolaterally to the AOTu. However, it does not seem to be associated or connected with the AOTu but exclusively interconnects the lobulae (Lo) of both brain hemispheres, in which it presents wide arborizations. Abbreviations are as defined in Figures 1 and 2 .

neurons shows the existence of different fascicles of fibers and arborizations into the four AOTu compartments (Fig. 4D,E). Despite their different trajectories, both tracts (vITT and mITT) are exclusively restricted to the AOTus and do not seem to receive any additional inputs. Thus, apart from their different paths, the only major difference between the two tracts is that mITT neurons have some very thin collaterals in the central brain (Fig. 4C). These fine collaterals appear to be output regions, given their minute diameter (Fig. 4C, inset) compared with that of the main axons.
The A5-2 neuron provides mushroombody input to the AOTu

Dextran-coupled dye injection into the AOTu also labeled an individual neuron with a very thick dendritic arbor in the vertical lobe of the ipsilateral mushroom body (Figs. $2 B, 5 A, B$ ). This neuron has been described previously in detail by Rybak and Menzel (1993) and is referred to as the A5-2 neuron (Fig. 5). It has a large cell body situated close to the inner side of the vertical lobe of the contralateral mushroom body (Fig. $5 A, C$ ) and shows putative axonal collaterals projecting alongside the ventral margin of the mushroom-body vertical lobe, farther into ventral and medial protocerebral neuropils and into the AOTu (Fig. 5A-C).

Thin axonal branches of the A5-2 neuron project toward the anterior surface of the ventral protocerebrum and penetrate the inner side of the AOTu, sending fine collaterals into the MU-DL and MU-VL (Fig. 5C,D). Dextran-coupled dye injection into the ventral part of the mushroom-body vertical lobe (Fig. $5 C$ ) labeled a network of fine A5-2 axon collaterals in the AOTu (Fig. 5D). Processes of these terminal branches typically form numerous blebby varicosities in both lobes of the AOTu major unit (Fig. 5D). Similar fine blebby collaterals also invade other parts of the lateral and medial protocerebrum (Fig. 5C), although innervation in the AOTu is much denser than in other parts of the protocerebrum. All in all, the overall appearance of the A5-2 neuron suggests that it receives input in the mushroom body and innervates ventrolateral and ventromedial regions of the protocerebrum, in particular the MU of the AOTu.

\section{The TALT conveys information from the AOTu to the median protocerebrum}

The AOTu sends putative output fibers to the median protocerebrum through the TALT (Figs. 1E, 2B), which exits the AOTu on its posterodorsal side (Fig. 6). Fibers of the TALT run laterally around the vertical lobe together with the mITT (Figs. $4 A, B, 6$ ) but turn posteriorly around the ipsilateral mushroom-body vertical lobe and pedunculus (Fig. $6 \mathrm{~A}$ ) before entering the LAL. Specific tracer injections into the LAL (Fig. 6A) reveal different types of neurons with distinct dendritic ramifications in the AOTu (Fig. 6B, C). Figure $6 B$ shows a small number of fibers whose dendrites form a dense network of arborizations in the MU-VL and in the LU. A different preparation (Fig. 6A, C) shows a large number of labeled TALT fibers, including fibers with a large diameter that present conspicuous thick branches on the most posterior side of the MU and the LU. No varicosities were visible inside the AOTu in these preparations, and the general 
appearance of these neurons suggests that they are presynaptic in the LAL (i.e., they represent output from the AOTu to the LAL).

Tracer injections into the AOTu revealed different types of output neurons with distinct arborization patterns and terminals in the LAL (Fig. 7). Although some AOTu output neurons of the TALT arborize throughout the ventral LAL (Fig. $7 A$ ), other neurons have terminals in confined areas of the LAL surrounding the central body and featuring unusually large knob-shaped structures (Fig. 7B-E). These large irregular terminals (Fig. $7 D, E$ ) are more pronounced in some TALT neurons than in others. We typically observed a high concentration of such microglomerular terminals forming a grape-like cluster ventrolaterally with respect to the lower unit of the central body (Fig. 7 B, D). More laterally, another set of neurons also presents such large microglomerular terminals in the vicinity of the central body (Fig. 7C,E). These terminals are supplied by very thin axon collaterals but are as large as small cell bodies: up to $4 \mu \mathrm{m}$ wide and $6 \mu \mathrm{m}$ long. Other TALT neurons that terminate in the most ventral part of the LAL [previously referred to as the ventral body (Milde 1988)] usually do not present this kind of terminals (Fig. 7A).

The anatomy of the LAL in honeybees has never been described in much detail, but a few studies identified this brain region as a source of different types of neurons sending input to the central body (Homberg, 1985; Milde, 1988). In addition to AOTu output neurons, our tracer injections into the LAL revealed a high number of neurons projecting into the upper and lower division of the central body (Fig. 6A), thus suggesting that visual information carried from the AOTu by TALT neurons might contribute to neural processing in the central body.

\section{Visually evoked calcium signals in AOTu inter-tubercle neurons}

Our anatomical characterization of the AOTu has shown that visual input from the medulla and lobula is segregated in the AOTu (Fig. 3D, E, H,I) (schematized in Fig. 10) so that visual signals from the dorsal and ventral parts of the bee eye appear to be differentially processed in distinct subcompartments (Figs. $1 B-E, 2 A$ ). Is the apparent dorsoventral segregation of visual input to the AOTu maintained at the output stage of the AOTu? To answer this question, we established a preparation (Fig. 8A) to record the responses of inter-tubercle neurons at a network level (output neurons from the AOTu running in the vITT and mITT) (Fig. 4) using in vivo calcium imaging.
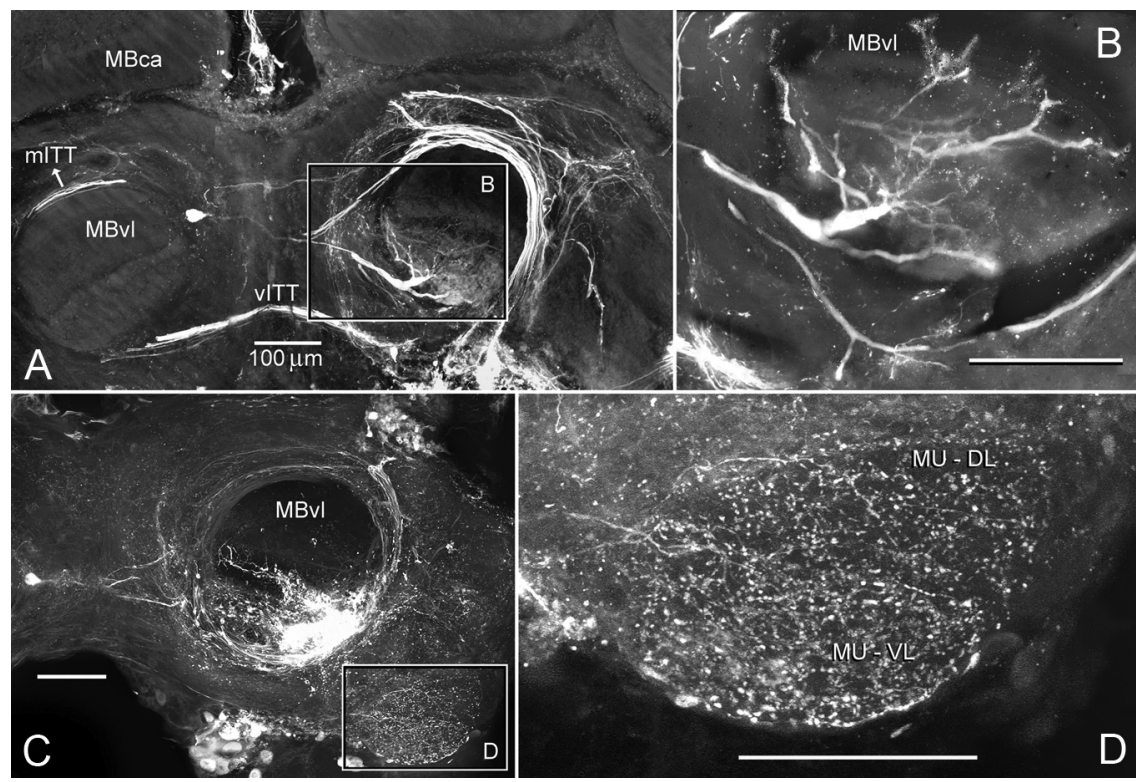

Figure 5. A mushroom-body extrinsic neuron that terminates in the A0Tu. $A$, Dextran-coupled dye injection into the A0Tu typically labels the A5-2 neuron (Rybak and Menzel, 1993) with cell body situated close to the inner side of the contralateral MBvl. This large neuron presents a very thick dendritic arbor in the ventral part of the ipsilateral MBvl $(\boldsymbol{B})$. $\boldsymbol{C}$, Dextran-coupled dye injection into the ventral part of the mushroom-body vertical lobe labels a network of fine A5-2 axon collaterals in the A0Tu. Areas boxed in $\boldsymbol{A}$ and $\boldsymbol{C}$, respectively, are enlarged in $\boldsymbol{B}$ and $\boldsymbol{D}$, respectively. $\boldsymbol{D}$, Thin axons originating from the $A 5-2$ neuron penetrate the inner side of the AOTu and send two main axonal branches into the MU-DL and MU-VL that end in numerous blebby varicosities. Abbreviations are as defined in Figures 1 and 2. Scale bars, $100 \mu \mathrm{m}$.
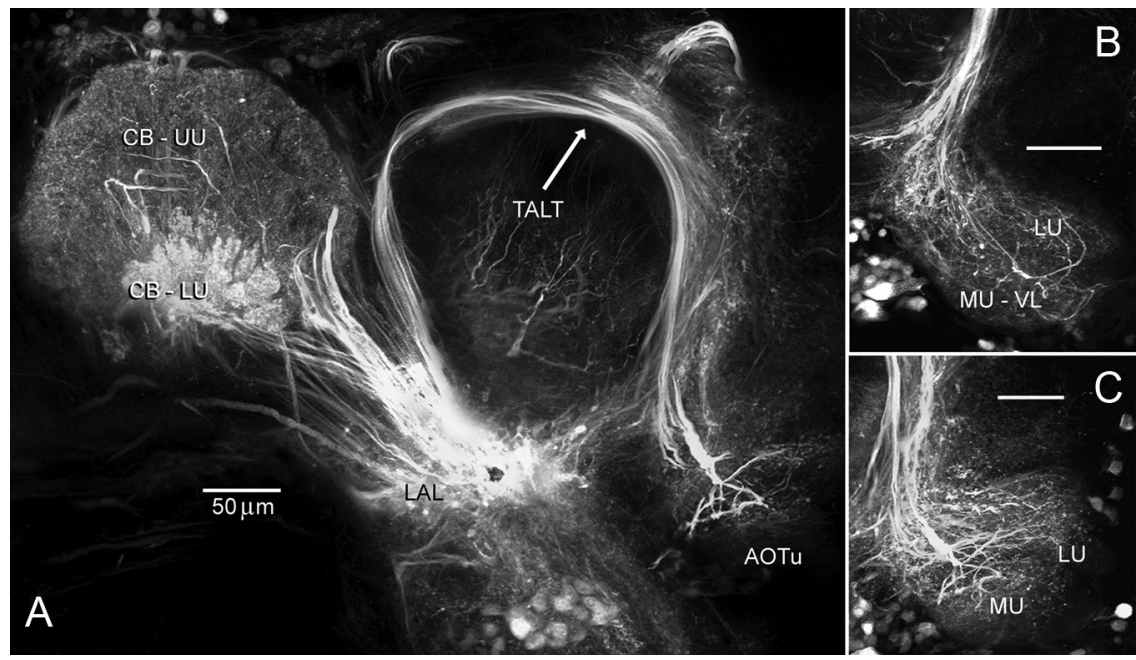

Figure 6. AOTu output to the median protocerebrum through the TALT. $A$, Localized tracer injection into the LAL reveals neurons of the TALT with dendritic ramifications in the AOTu, as well as neurons connecting the $L A L$ with the lower and upper units of the central complex (CB-UU and $C B-L U)$. Fibers of the TALT run laterally and turn around the ipsilateral MBvl before entering the LAL. $\boldsymbol{B}, \boldsymbol{C}$, Frontal sections of the AOTu showing different types of TALT neurons with arborizations in the AOTu. $\boldsymbol{B}$, TALT fibers whose dendrites form a dense network of arborizations in the MU-VL and in the LU. C, A composite of all the confocal sections from the AOTu stained in $\boldsymbol{A}$ shows a large number of labeled TALT fibers, including fibers that present conspicuous thick branches on the posteriormost side of the MU and the LU. Abbreviations are as defined in Figures 1 and 2. Scale bars, $50 \mu \mathrm{m}$.

We took advantage of the fact that highly reproducible retrograde staining of ITTs and their dendritic arborizations in one AOTu can be obtained by injection of a dextran tracer into the contralateral AOTu (Fig. 4B). This allowed us to focus on calcium responses in the ipsilateral $\mathrm{AOTu}$ (opposite to the dextran injection side) to determine how AOTu output neurons leaving the ipsilateral AOTu to the contralateral AOTu respond to visual information from the dorsal, lateral, and ventral parts of the visual field of the ipsilateral eye (Fig. $8 B$ ). 

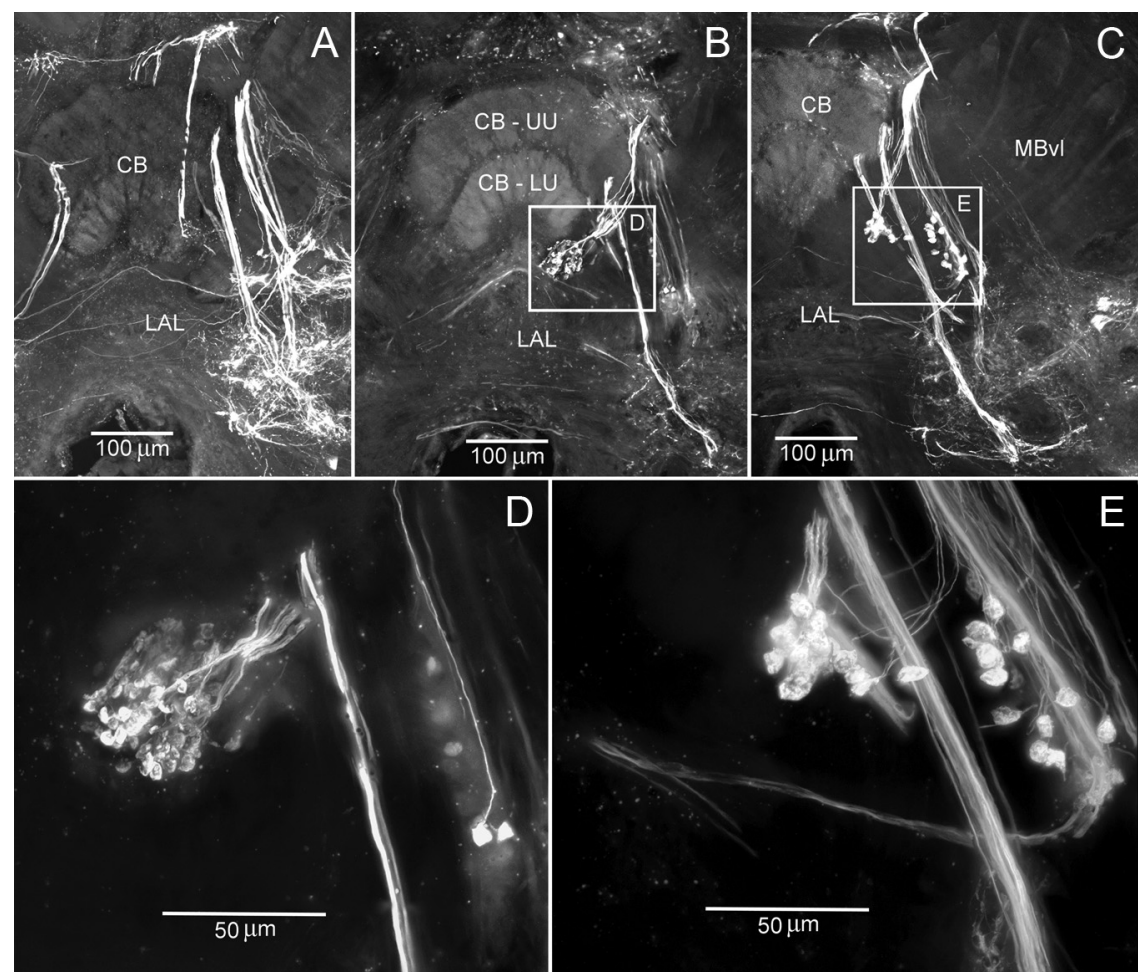

Figure 7. Dextran injections into the AOTu revealing conspicuous types of TALT axon terminals in the LAL. A, AOTu output neurons of the TALT widely arborizing the ventral LAL. $\boldsymbol{B}$, High concentration of microglomerular terminals forming a grape-like cluster ventrolaterally with respect to the lower unit of the central complex (CB-LU). C, Another set of neurons also presenting such large microglomerular terminals in the vicinity of the $C B . \boldsymbol{D}, \boldsymbol{E}$, Enlargements of the microglomerular clusters in preparations $\boldsymbol{B}$ and $C$, respectively. Although supplied by very thin axon collaterals, these microglomerular terminals are as large as small cell bodies: up to $4 \mu \mathrm{m}$ wide and $6 \mu \mathrm{m}$ long. Abbreviations are as defined in Figures 1 and 2. CB-UU, Upper unit of the central complex.

activated both MU units (Fig. 8C). To provide a quantitative analysis of spatial activation patterns in the AOTu, we focused on pixels that were significantly activated, i.e., that showed a signal above noise level (see Materials and Methods), and thereby obtained activity maps corresponding to dorsal, lateral, and ventral stimulations of the ipsilateral compound eye. Figure $9 A$ (left) presents such maps recorded in one animal during three dorsal, lateral, or ventral stimulations.

To determine to what extent each visual stimulus evoked reproducible calcium signals, density maps were calculated in a color code indicating the number of times each pixel was activated during the three identical stimulations (Fig. 9A, middle). Pixels that were systematically activated during each stimulation were defined as reliably activated and are indicated in white (Fig. 9A, middle). Spatial distribution of reliably activated pixels revealed a clear segregation of the signals in the DL and VL part of the MU depending on the area of stimulation of the ipsilateral compound eye. Such a segregation became most conspicuous when overlay maps were obtained (Fig. 9A, right) by establishing which reliable pixels (in white) were activated by two different visual-field stimulations [i.e., dorsal (magenta) and lateral (blue); ventral (green) and lateral (blue); or dorsal (magenta)

Because our recordings used a conventional fluorescence microscope, which is best suited for imaging of superficial tissue, calcium signals could only be recorded in the anterior part of the $\mathrm{AOTu}$, in which the MU is most prominent (Figs. 1C, 4D). All three white LED arrays presented in the dorsal, lateral, or ventral parts of the visual field (Fig. $8 \mathrm{~B}$ ) elicited clear calcium signals in the AOTu ( $n=10$ bees) (see two examples in Fig. $8 C$ ), with consistent and similar phasic-tonic time courses (Fig. $8 D$ ). For all visual stimulations, the amplitude of calcium responses was significantly higher than that of control recordings without any light (Fig. $8 E$ ) (Friedman's test: $\chi^{2}=21.72$, df $=3, p<0.0001$; Wilcoxon's matched-pairs test: $Z=2.80, p<0.01$ in all cases). The mean amplitude of laterally evoked signals was significantly higher than that of ventrally evoked signals (Fig. $8 E)(Z=2.21$, $p<0.02$ ), whereas responses to dorsal light did not differ from lateral or ventral light stimulations (Fig. $8 E$ ) (dorsal vs lateral: $Z=1.78$, NS; dorsal vs ventral: $Z=0.97$, NS). Thus, for the first time, our preparation allowed recording consistent and reproducible calcium responses in the honeybee AOTu during visual stimulation of the compound eye. This allowed us to address the question of whether distinct spatial stimulations of the compound eye result in distinct spatial activation responses in the $\mathrm{AOTu}$, as predicted from our neuroanatomical characterizations.

\section{Spatial coding in the AOTu}

Stimulation of distinct parts of the visual field induced different activation patterns in the AOTu (Fig. 8C), consistent with the segregation of input to the MU-VL and MU-DL. Dorsal eye stimulation activated mostly the MU-VL and ventral stimulation activated principally the MU-DL, whereas lateral stimulation and ventral (green)]. The high specificity of activation maps obtained during different spatial stimulations of the compound eye can be observed in terms of the low proportion of common pixels (white pixels in Fig. 9A, right) relative to all reliably activated pixels (all pixels in Fig. 9A, right). This specificity can be quantified by computing an index of the relative overlap between any two stimulation maps, as the number of pixels in common divided by the number of pixels activated by any of the two stimuli (see Materials and Methods). A value of 1 indicates a full coincidence between these two variables, thus reflecting no spatial segregation of activation maps; in contrast, values close to 0 correspond to a clear segregation (no or few pixels in common) of activation maps. The index was calculated for (1) stimulations of the same part of the visual field (Overlap same, i.e., dorsal vs dorsal, ventral vs ventral, or lateral vs lateral) or (2) stimulations of different parts of the visual field (Overlap different, i.e., dorsal vs ventral, dorsal vs lateral, or ventral vs lateral). Figure $9 B$ shows that the average Overlap $_{\text {same }}$ obtained for 10 bees was significantly higher than the average Overlap $_{\text {different }}(Z=2.80, p<0.01)$, thus indicating that stimulations of different regions of the compound eye induced spatially segregated activation maps at the level of the MU of the AOTu.

The activity patterns evoked by stimulation of different parts of the visual field were highly reproducible within and among individuals ( $n=10$ bees). In all bees, dorsal and ventral eye stimulations induced activity mostly in the MU-VL and MU-DL, respectively (Fig. 9A, $C$, schematized in Fig. 10). Figure 9C illustrates well this spatial segregation in four animals whose overlay maps of reliably activated pixels during dorsal (magenta) and ventral (green) stimulations reveal very few common pixels 
A

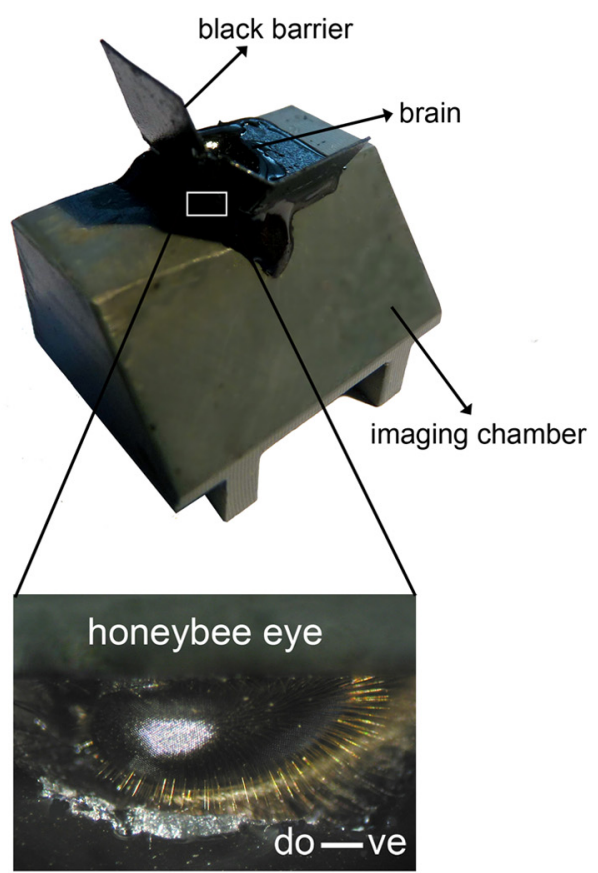

C
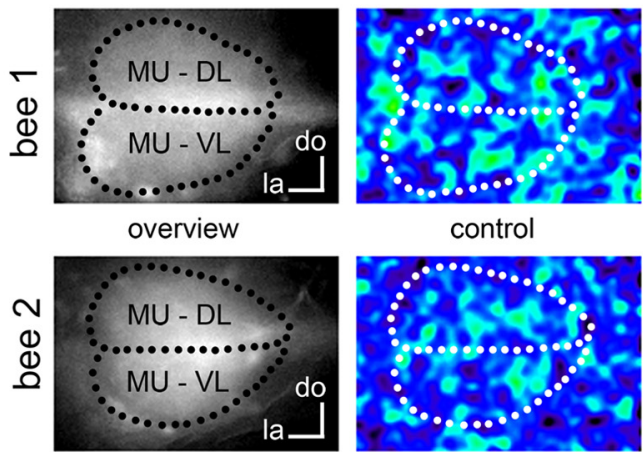

control

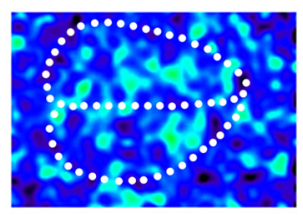

D

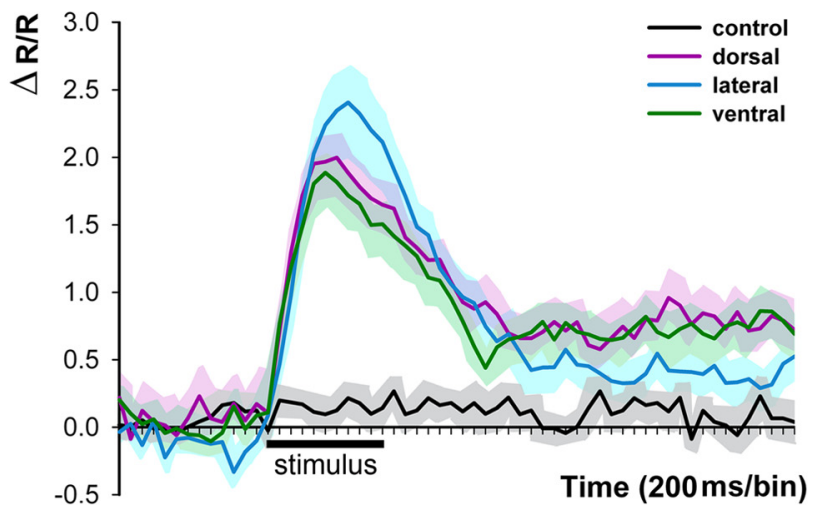

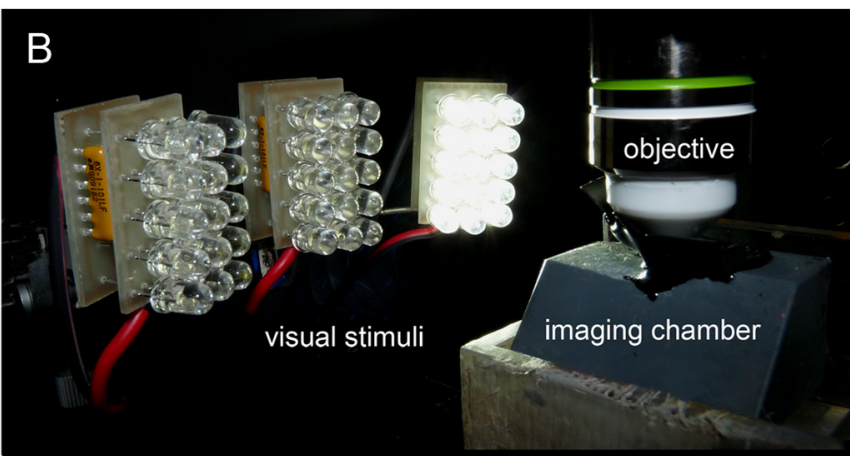

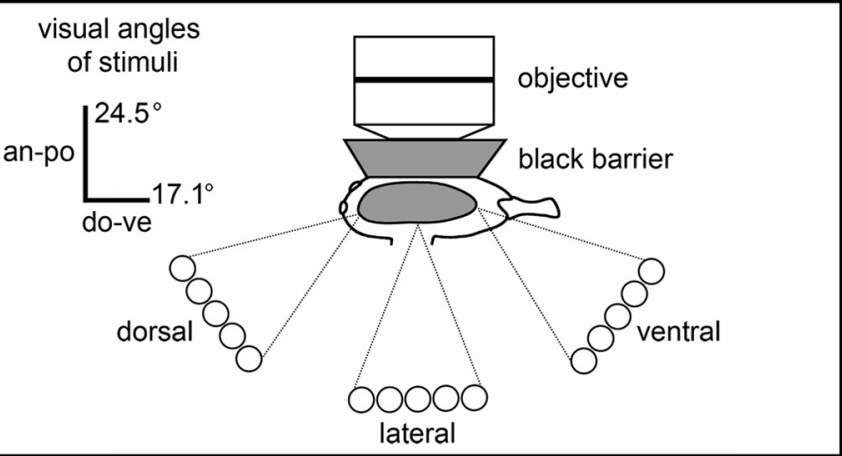

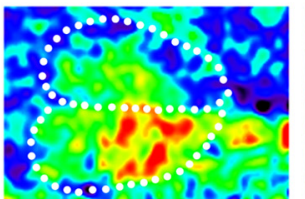

dorsal

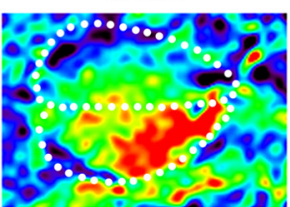

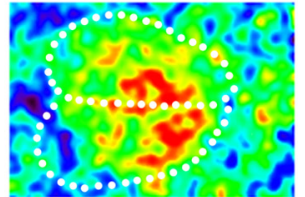

lateral

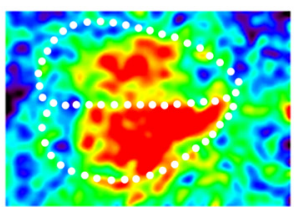

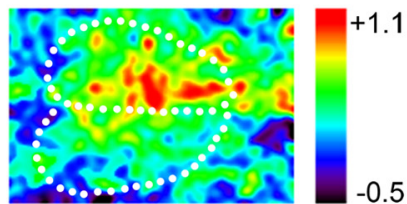

ventral

$\Delta R / R$

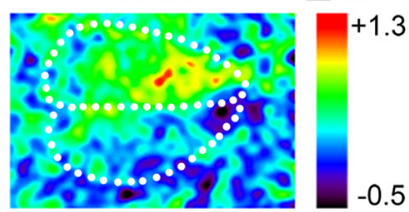

E

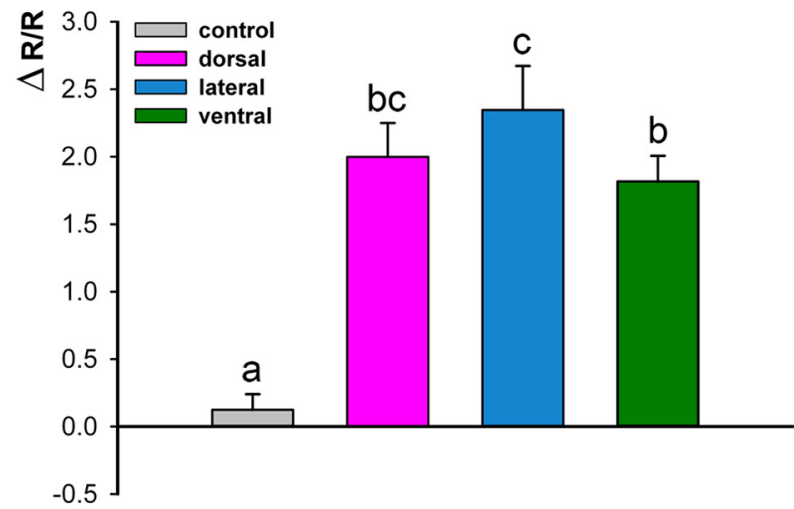

Figure 8. Optical recordings of calcium signals from inter-tubercle neurons in the honeybee AOTu. $\boldsymbol{A}$, Bees are placed individually in opaque recording chambers. The brain area is optically isolated from the compound eye area in which visual stimuli are given, using opaque barriers glued to the bee head with custom black wax. $\boldsymbol{B}$, Visual stimulation is produced by three rectangular LED arrays $(3 \times 5$ LEDs each) disposed in a half-circle to stimulate different parts of the visual field (dorsal, lateral, or ventral) of the bee right eye. The center of each array is placed at a distance of $6 \mathrm{~cm}$ from the eye, corresponding to an angular subtense of $24.5^{\circ}$ (anteroposterior axis) and $17.1^{\circ}$ (dorsoventral axis) at the bee eye. C, False color-coded activation maps (percentage change in $340 \mathrm{~nm} / 380$ $\mathrm{nm}$ fluorescence ratio; see Materials and Methods) obtained in the right AOTu for two different animals (bee 1 and bee 2). The contours of MU-DL and MU-VL (dashed lines) are clearly visible on the AOTu anterior surface after fura-2 dextran staining of inter-tubercle neurons (overview). Dendrites of inter-tubercle neurons in the A0Tu show calcium signals in response to light stimulations but not to dark controls. Stimulations of all three parts of the visual field clearly activate the A0Tu. D, Time course of fura- 2 calcium signals (percentage change in fluorescence ratio, average of three presentations for each bee, $n=10$ bees) measured in the MU of the A0Tu for stimulation of dorsal, lateral, or ventral parts of the visual field and for the dark control. $\boldsymbol{E}$, Mean amplitude of activation (calculated at $4 \mathrm{~s}$ ) for stimulations of dorsal, lateral, or ventral parts of the visual field and dark control in 10 bees. Different letters indicate significant differences in Wilcoxon's matched-pairs tests. do-ve, Dorsoventral; an-po, anteroposterior; do, dorsal; la, lateral. 
A
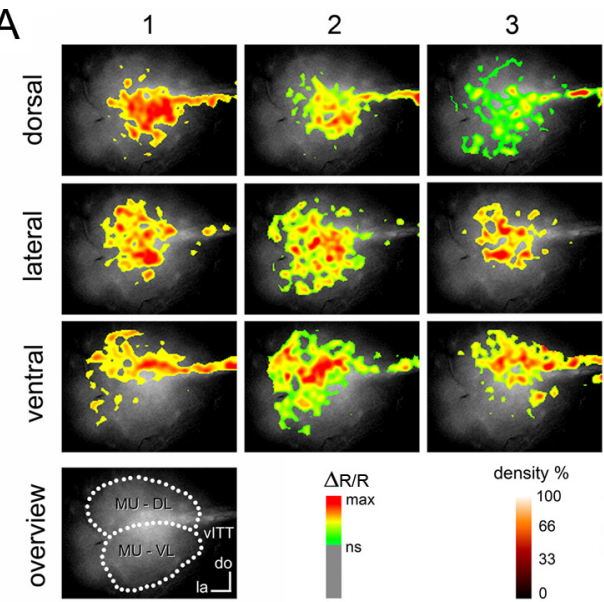

B

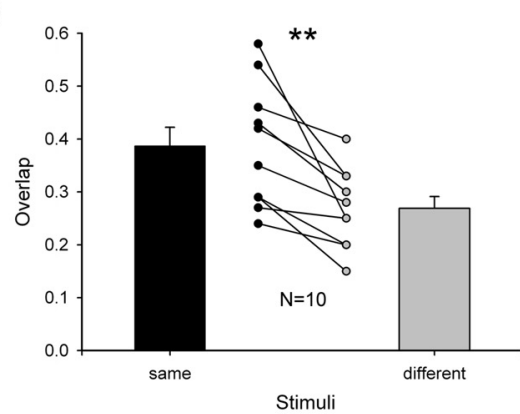

D

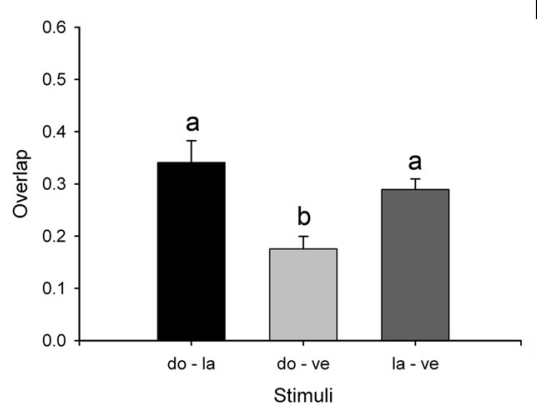

$\mathrm{E}$

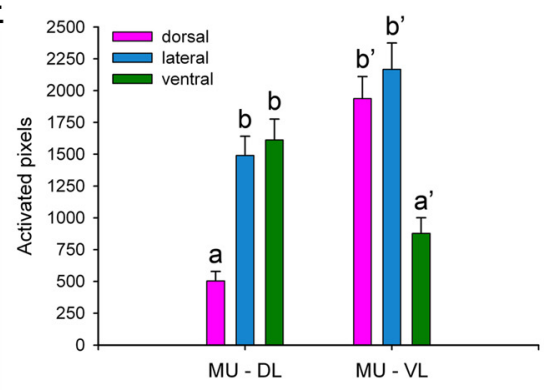

Figure 9. Spatial coding in the honeybee AOTu. $A$, Example of activated pixels for three repetitions of dorsal, lateral, or ventral stimulations in the MU of the AOTu (overview). Significantly activated pixels are overlaid on a fluorescence photograph of the A0Tu according to their amplitude of activation $(\Delta R)$ in false color. Density maps indicate the reliability of activated pixels: colors from dark red to white indicate pixels activated by one, two, or the three presentations of each stimulus. Overlay maps represent reliable pixels activated by the three stimulations of one part of the visual field in one color and the other part in another color (dorsal in magenta, lateral in blue, and ventral in green). Pixels activated by stimulations of both parts of the visual field are represented in white. These maps show good reproducibility of activated pixels for the same part of the visual field (density) and a segregation of activated pixels for different parts of the visual field (overlay, especially dorsal vs ventral). $\boldsymbol{B}$, Comparison of the mean overlap between any two presentations of the same or of different stimuli in 10 bees. Activation patterns in the AOTu show clear spatial specificity, because presentations of the same stimulus present a higher overlap than presentations of different stimuli (Wilcoxon's signed-rank test). C, Four examples of the striking segregation between dorsal and ventral stimulations. White arrows in bee 1 and bee 4 indicate a dorsoventral segregation at the level of the axonal tract of vITT neurons (see also bee shown in $\boldsymbol{A}$ ). $\boldsymbol{D}$, The overlap index between dorsal and ventral stimuli (do-ve) is significantly lower than that between lateral and dorsal stimuli (do-la) or between lateral and ventral stimuli (la-ve) as shown by Wilcoxon's signed-rank test. $\boldsymbol{E}$, Mean number of activated pixels in the MU-DL or MU-VL for stimulations of the dorsal, lateral, and ventral eye in 10 bees. In the MU-DL, ventral and lateral stimulations activated a significantly higher number of pixels than dorsal stimulation, whereas in the MU-VL, dorsal and lateral stimulations activated a higher number of pixels than ventral stimulation (Wilcoxon's signed-rank test).

(white). Calcium signals recorded from the vITT tract at the point where it leaves the MU were also often segregated in the overlay maps between dorsal and ventral stimulation (see the thin lines of activation on the right side of Fig. $9 A$, right panel and of Fig. $9 C$, bee 1 and bee 4 , white arrows), supporting the dorsoventral segregation of information conveyed by inter-tubercle neurons to the opposite AOTu. In agreement with the above observations, the overlap index between dorsal and ventral stimuli $\left(\right.$ Overlap $\left._{\text {do-ve }}\right)$ (Fig. 9D) was significantly lower than that between lateral and dorsal stimuli $\left(\right.$ Overlap $\left._{\text {do-la }}\right)$ or between lateral and ventral stimuli (Fig. 9D) (Friedman's test: $\chi^{2}=15.20, \mathrm{df}=2, p<0.001 ;$ Overlap $_{\mathrm{do}-\mathrm{ve}}$ vs Overlap $_{\text {do-la: }}: Z=2.80, p<0.01$; Overlap $_{\text {do-ve }}$ vs Overlap la-ve $Z=2.80, p<$ $0.01)$. The overlap indices between lateral and dorsal stimuli (Overlap do-la) and between lateral and ventral stimuli $\left(\right.$ Overlap $\left._{\text {la-ve }}\right)$ did not differ significantly (Fig. 9D) (Overlap do-la vs Overlap la-ve: $Z=1.17, \mathrm{NS}$ ).

To better analyze the distribution of calcium signals evoked by dorsal, lateral, or ventral visual stimuli within the MU-DL or MU-VL, we quantified the number of activated pixels within each of these AOTu subunits. In the MU-DL, ventral stimulation activated a significantly higher number of pixels than dorsal stimulation (Fig. 9E) (Friedman's test: $\chi^{2}=12.20, \mathrm{df}=2, p<0.01$; ventral vs dorsal: $Z=2.80, p<0.01$ ). Conversely, in the MU-VL, dorsal stimulation activated a higher number of pixels than ventral stimulation (Fig. 9E) (Friedman's test: $\chi^{2}=15.8$, $\mathrm{df}=2, p<0.001$; dorsal vs ventral: $Z=$ $2.80, p<0.01)$. Lateral stimulations activated the same amount of pixels as ventral stimulations in the MU-DL $(Z=0.56, \mathrm{NS})$ and as dorsal stimulations in the MU-VL $(Z=1.78, \mathrm{NS})$.

Our results show, therefore, that the two different subunits of the MU (MU-DL and MU-VL) represent distinct spatial visual information, in such a way that the MU-VL responds mainly to visual stimulations of the dorsal region of the compound eye, whereas the MU-DL responds mainly to stimulations of the ventral region of the compound eye (schematized in Fig. 10). Such dorsoventrally segregated output responses reflect to some extent the segregation of visual input to the AOTu uncovered by our neuroanatomical analyses (Fig. 3D,E,H,I). However, one should keep in mind that the anatomical tracing experiments detailed the organization of direct input from the (ipsilateral) optic lobe, whereas the optophysiological data reveal activity patterns of output neurons projecting to the contralateral AOTu (Fig. 9A,C). Figure 10 schematizes how dorsoventral information is organized at both the input (anatomical data) and output (physiological data) levels of this AOTu circuitry.

\section{Discussion}

In the present work, we analyzed the internal organization and neuronal connections of the AOTu in the honeybee. In addition, we established a novel protocol for optophysiological recordings 


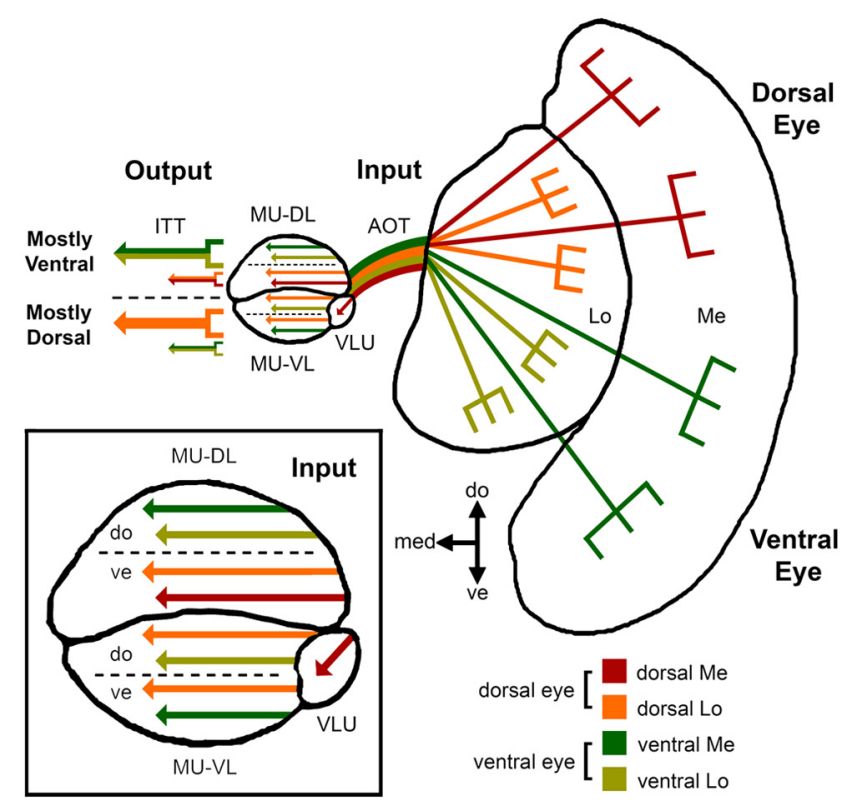

Figure 10. Schematic representation of dorsoventral visual information flow both at the input (anatomical data, see Fig. 3) and at the output levels (physiological data, see Fig. 9) of the AOTu circuitry. Input, From medulla (Me) and lobula (Lo) to the AOTu via the AOT. The input pattern is enlarged in the boxed inset. Ventral eye: Ventral medulla neurons (ventral Me, dark green) project to the MU-VL (chiefly to its ventral part) and to the dorsal part of the MU-DL; ventral lobula neurons (ventral Lo, light green) project to the dorsal parts of the MU-DL and MU-VL. Dorsal eye: Dorsal medulla neurons (dorsal Me, red) only project to the ventral part of the MU-DL and to the VLU; dorsal lobula neurons (dorsal Lo, orange) project to the ventral part of the MU-DL and to the whole MU-VL. Output: From the ipsilateral AOTu to the contralateral AOTu via ITT. Ventral eye: Output inter-tubercle neurons in MU-DL are mostly activated by ventral eye stimulation. Dorsal eye: Output inter-tubercle neurons in MU-VL are mostly activated by dorsal eye stimulation. Thus, functionally, inter-tubercle output neurons in MU-DL seem to be mainly connected to the input neurons from the ventral eye (dark and light green arrows) to this compartment, whereas output neurons in MU-VL seem to be mainly connected to the input neurons from the dorsal eye to this compartment (orange arrows).

of visual circuit activity in the honeybee brain and studied the responses of AOTu interneurons during stimulation of distinct eye regions. Our anatomical data reveal an intricate compartmentalization and connectivity, which together with spatially dependent physiological responses, point toward an involvement of the AOTu in the processing of dorsoventrally organized visual information.

\section{The neural architecture of the AOTu}

Our analyses showed that the honeybee AOTu is composed of four compartments: the MU-VL and the MU-DL that together constitute a major unit, and two smaller units placed posterior to the MU, the VLU and the LU. We found retinotopic small-field input to the MU, with dorsal and ventral parts of the medulla and lobula differentially supplying MU-VL and MU-DL. In contrast, the VLU only receives input from the dorsal medulla. Input from the mushroom body supplies the MU exclusively. The LU, conversely, does not seem to receive input from the optic lobe or mushroom body but is connected to the contralateral AOTu and to the LAL. We cannot exclude, however, the possibility of having missed in our mass fillings some specific optic lobe input to the LU. Inter-tubercle neurons innervate all AOTu compartments in both brain hemispheres. Each AOTu receives from and provides to the contralateral AOTu dorsoventral segregated information. Distinct types of output neurons connecting the AOTu with the LAL appear to originate from different AOTu compartments, but the organization of dorsoventral information in these neurons remains to be clarified by additional studies. Our results show that some level of segregation between dorsal and ventral eye information occurs in the AOTu and point toward a specialization of certain AOTu compartments for this segregated spatial processing.

\section{Input-output circuits in the AOTu}

Input from the optic lobe runs via the AOT, which contains two main neural types: (1) transmedullary neurons and (2) lobula columnar neurons. Dual supply from the medulla and lobula to the AOTu was also described in butterflies (Strausfeld and Blest, 1970), flies (Strausfeld and Nässel, 1981), and locusts (Homberg et al., 2003). In flies, the two types of small-field neurons projecting to the AOTu have dendrites confined to specific areas of medulla or lobula, supposedly conveying information from circumscribed areas of the retina (Strausfeld, 1970; Strausfeld and Okamura, 2007). Our retrograde staining shows the same arrangement in bees (Figs. 3, 4A), thereby providing an ordered input necessary for retinotopic organization. The honeybee $\mathrm{AOTu}$ also receives central input from the ipsilateral mushroom body via the A5-2 neuron (Rybak and Menzel, 1993), suggesting that the AOTu might be involved in higher-order, cognitive processing. The documented role of the mushroom- body extrinsic neurons in learning, memory, and multimodal perception (for review, see Menzel, 1999; Menzel and Giurfa, 2001; Giurfa, 2007) suggests the possible involvement of the AOTu in these phenomena.

Output fibers from the AOTu project mostly to the LAL via the TALT, which comprises different neurons with distinct arborization patterns in both the AOTu and the median protocerebrum. Some show large irregular terminals in the LAL, in which they form two main clusters, a grape-like cluster ventrolaterally to the central body and another cluster located more laterally. In locusts, similar giant terminals are concentrated in two LAL subcompartments, the lateral triangle and the median olive (Homberg et al., 2003), which correspond to the localizations found in the bee. These terminals provide synaptic input to tangential neurons of the central body of the locust (Träger et al., 2008). TALT neurons in the honeybee might thus provide a similar input at the level of the central body.

Additional input and output circuits are provided by the vITT and mITT pathways, which interconnect the AOTus of both hemispheres, thus pointing to a major role in the comparison of information from the two compound eyes. Inter-tubercle neurons in the honeybee arborize in all four AOTu compartments, thus connecting the whole AOTu structures of both brain hemispheres. These neurons thus represent a rich substrate for studying the functional role of distinct AOTu compartments, a possibility we have successfully exploited in our calcium imaging recordings.

\section{AOTu function in a physiological and behavioral context}

Knowledge of AOTu function in bees was scarce, and the few previous recordings obtained from isolated neurons pointed to a possible involvement of the AOTu in the processing of chromatic information (Paulk et al., 2008, 2009). Here we provide the first functional data on AOTu neural populations. Our in vivo recordings of inter-tubercle neurons revealed that light stimuli presented in different parts of the visual field elicited distinct spatial activity patterns, with a clear dorsoventral segregation of calcium signals. Stimulation of the dorsal region of the visual field induced activity mostly in the MU-VL, whereas stimulation of the ventral region induced activity mostly in the MU-DL. The segre- 
gation of dorsal and ventral stimulus representation seems to occur not only in the main body of the MU but also in the axons of inter-tubercle neurons, which showed differential activation between dorsal and ventral stimuli. Despite the coherence between dorsoventral segregation in the input from the optic lobe to the MU (anatomical data) and in the output from the MU to the contralateral AOTu (physiological data), some mismatch in the neural organization seems to exist between the input and the output level. The pattern of dorsoventral segregation observed in the MU does not seem to be exactly the same from one level to the other (Fig. 10). This change may be related to selective connections between these input and output neurons, so that intertubercle output neurons in MU-VL would mainly connect to the input neurons from the dorsal eye to this compartment, whereas output neurons in MU-DL would mainly connect to the input neurons from the ventral eye to this compartment (Fig. 10). More complex connections between input and output neurons, as well as lateral inhibition within the AOTu, may also be involved in this reorganization of dorsoventral information. Additional studies focusing on distinct individual neurons in this circuitry may contribute to elucidating this issue.

The VLU of the honeybee AOTu shows remarkable similarity with the lower unit of the locust AOTu (Homberg et al., 2003), which processes polarized light information and conveys it via the LAL to the central body (Pfeiffer et al., 2005; Heinze and Homberg 2007, 2009; Kinoshita et al., 2007). Both are positioned ventrodorsally and posterior to the more prominent AOTu unit (MU in honeybees; upper unit in locusts) and receive input from the dorsal medulla. Thus, the honeybee VLU may be functionally homologous to the locust lower unit. Furthermore, the upper unit of the locust AOTu, whose function is as yet unknown, shares remarkable similarities with the MU of the honeybee $\mathrm{AOTu}$. Both constitute the largest AOTu volume and are composed of two subunits (outer and inner lobes in locusts; MU-VL and MU-DL in honeybees). Our results suggest a spatial information content at the MU inter-tubercle output, and likewise, upper unit interneurons in locusts respond in a strongly stimulus position-dependent manner (Pfeiffer and Homberg, 2002; Pfeiffer et al., 2005; Kinoshita et al., 2007). We thus believe that this bi-lobed major AOTu compartment serves similar spatial processing functions in honeybees and locusts. The central body of insects presumably participates in polarized light coding, visual pattern recognition, spatial orientation, right-left maneuvering, and motor control (Strauss, 2002; Vitzthum et al., 2002; Liu et al., 2006; Wang et al., 2008). The AOTu output neurons may indirectly (via the LAL) provide the central body with the spatial information necessary for these tasks.

Behavioral experiments show remarkable asymmetries in visual performances involving the dorsal or ventral honeybee eye regions (see below). The whole dorsal retina (not only the dorsal rim area) seems to participate in the processing of sky cues such as polarization, sun position and azimuth, and spectral gradients (Menzel and Snyder, 1974; Menzel and Lieke, 1983; Rossel and Wehner, 1986; Lehrer, 1998). The MU-VL and the VLU, which are supplied by dorsal eye information, may therefore participate in the central processing of skylight visual cues, in particular, polarization and UV light because both provide navigation cues for identifying the anti-solar hemisphere of the sky dome (Wehner and Rossel, 1985). Interestingly, more UV photoreceptors are proportionally found in both the dorsal rim area and the dorsal retina of the honeybee (Wakakuwa et al., 2005). Furthermore, both AOTu compartments should be able to respond to greenreceptor information, which provides a navigating bee with spec- tral information directly related to the sun (Wehner and Rossel, 1985).

The ventral eye region of honeybees is involved in color, shape, pattern, and spatial frequency detection and discrimination (Wehner, 1972; Anderson, 1977; Giger and Srinivasan, 1997; Lehrer 1998, 1999; Giurfa et al., 1999). The MU-DL of the honeybee AOTu, which is more activated by ventral eye stimulation, may thus intervene in the processing of these visual cues, which have been shown to recruit all three types of photoreceptors: UV, blue, and green sensitive. Our novel functional imaging technique will be a key tool for studying the role of different AOTu neural populations for the processing of different visual stimuli categories, such as color, achromatic patterns, or motion.

\section{References}

Anderson AM (1977) Parameters determining the attractiveness of stripe patterns in the honeybee. Anim Behav 25:80-87.

Deisig N, Giurfa M, Lachnit H, Sandoz JC (2006) Neural representation of olfactory mixtures in the honeybee antennal lobe. Eur J Neurosci 24: 1161-1174.

Deisig N, Giurfa M, Sandoz JC (2010) Antennal lobe processing increases separability of odor mixture representations in the honeybee. J Neurophysiol 103:2185-2194.

DeVoe RD, Kaiser W, Ohm J, Stone LS (1982) Horizontal movement detectors of honeybees: directionally-selective visual neurons in the lobula and brain. J Comp Physiol 147:155-170.

Douglass JK, Strausfeld NJ (1998) Functionally and anatomically segregated pathways in the lobula complex of a calliphorid fly. J Comp Neurol 396:84-104.

Ehmer B, Gronenberg W (2002) Segregation of visual input to the mushroom bodies in the honeybee (Apis mellifera). J Comp Neurol 451:362-373.

Galizia CG, Vetter RS (2005) Optical methods for analizing odor-evoked activity in the insect brain. In: Advances in insect sensory neuroscience (Christensen TA, ed), pp 349-392. Boca Raton, FL: CRC.

Giger A, Srinivasan M (1997) Honeybee vision: analysis of orientation and colour in the lateral, dorsal and ventral fields of view. J Exp Biol 200:1271-1280.

Giurfa M (2007) Behavioral and neural analysis of associative learning in the honeybee: a taste from the magic well. J Comp Physiol A Neuroethol Sens Neural Behav Physiol 193:801-824.

Giurfa M, Vorobyev M, Kevan P, Menzel R (1996) Detection of coloured stimuli by honeybees: minimum visual angles and receptor specific contrasts. J Comp Physiol A Neuroethol Sens Neural Behav Physiol 178:699-709.

Giurfa M, Zaccardi G, Vorobyev M (1999) How do bees detect coloured targets using different regions of their compound eyes. J Comp Physiol A Neuroethol Sens Neural Behav Physiol 185:591-600.

Hadley A (2010) CombineZP. Available at http://www.hadleyweb.pwp. blueyonder.co.uk.

Heinze S, Homberg U (2007) Maplike representation of celestial E-vector orientations in the brain of an insect. Science 315:995-997.

Heinze S, Homberg U (2009) Linking the input to the output: new sets of neurons complement the polarization vision network in the locust central complex. J Neurosci 29:4911-4921.

Hertel H, Maronde U (1987) The physiology and morphology of centrally projecting visual interneurons in the honeybee brain. J Exp Biol 133:301-315.

Hertel H, Schäfer S, Maronde U (1987) The physiology and morphology of visual commisures in the honeybee brain. J Exp Biol 133:283-300.

Homberg U (1985) Interneurons of the central complex of the bee brain (Apis mellifica L.). J Insect Physiol 31:251-264.

Homberg U, Hofer S, Pfeiffer K, Gebhardt S (2003) Organization and neural connections of the anterior optic tubercle in the brain of the locust, Schistocerca gregaria. J Comp Neurol 462:415-430.

Joerges J, Küttner A, Galizia CG, Menzel R (1997) Representations of odours and odour mixtures visualized in the honeybee brain. Nature 387:285-288.

Kinoshita M, Pfeiffer K, Homberg U (2007) Spectral properties of identified polarized-light sensitive interneurons in the brain of the desert locust Schistocerca gregaria. J Exp Biol 210:1350-1361. 
Labhart T (1980) Specialized photoreceptors at the dorsal rim of the honeybee's compound eye: polarizational and angular sensitivity. J Comp Physiol A Neuroethol Sens Neural Behav Physiol 141:19-30.

Labhart T, Meyer EP (2002) Neural mechanisms in insect navigation: polarization compass and odometer. Curr Opin Neurobiol 12:707-714.

Lehrer M (1998) Looking all around: honeybees use different cues in different eye regions. J Exp Biol 201:3275-3292.

Lehrer M (1999) Dorsoventral asymmetry of colour discrimination in bees. J Comp Physiol A Neuroethol Sens Neural Behav Physiol 184:195-206.

Liu G, Seiler H, Wen A, Zars T, Ito K, Wolf R, Heisenberg M, Liu L (2006) Distinct memory traces for two visual features in the Drosophila brain. Nature 439:551-556.

Mares S, Ash L, Gronenberg W (2005) Brain allometry in bumblebee and honeybee workers. Brain Behav Evol 66:50-61.

Menzel R (1974) Spectral sensitivity of monopolar cells in the bee lamina. J Comp Physiol A Neuroethol Sens Neural Behav Physiol 93:337-346.

Menzel R (1999) Memory dynamics in the honeybee. J Comp Physiol A Neuroethol Sens Neural Behav Physiol 185:323-340.

Menzel R, Giurfa M (2001) Cognitive architecture of a mini-brain: the honeybee. Trends Cogn Sci 5:62-71.

Menzel R, Lieke E (1983) Antagonistic color effects in spatial vision of honeybees. J Comp Physiol 151:441-448.

Menzel R, Snyder AW (1974) Polarized light detection in the bee, Apis mellifera. J Comp Physiol 88:247-270.

Milde JJ (1988) Visual responses of interneurones in the posterior median protocerebrum and the central complex of the honeybee Apis mellifera. J Insect Physiol 34:427-436.

Mobbs PG (1982) The brain of the honeybee Apis mellifera. The connections and spatial organization of the mushroom bodies. Philos Trans R Soc Lond B Biol Sci 298:309-354.

Paulk AC, Phillips-Portillo J, Dacks AM, Fellous JM, Gronenberg W (2008) The processing of color, motion, and stimulus timing are anatomically segregated in the bumblebee brain. J Neurosci 28:6319-6332.

Paulk AC, Dacks AM, Phillips-Portillo J, Fellous JM, Gronenberg W (2009) Visual processing in the central bee brain. J Neurosci 29:9987-9999.

Peitsch D, Feitz A, Hertel H, de Souza J, Ventura DF, Menzel R (1992) The spectral input systems of hymenopteran insects and their receptor-based colour vision. J Comp Physiol A Neuroethol Sens Neural Behav Physiol Neuroethol Sens Neural Behav Physiol 170:23-40.

Pfeiffer K, Homberg U (2002) Visual signal processing in neurons of the anterior optic tubercle of the locust, Schistocerca gregaria. Zoology 105 [Suppl V]:45.

Pfeiffer K, Kinoshita M, Homberg U (2005) Polarization-sensitive and light-sensitive neurons in two parallel pathways passing through the anterior optic tubercle in the locust brain. J Neurophysiol 94:3903-3915.
Ribi WA, Scheel M (1981) The second and third optic ganglia of the worker bee: Golgi studies of the neuronal elements in the medulla and lobula. Cell Tissue Res 221:17-43.

Rossel S, Wehner R (1986) Polarization vision in bees. Nature 323:128-131.

Rybak J, Menzel R (1993) Anatomy of the mushroom bodies in the honeybee brain: the neuronal connections of the alpha-lobe. J Comp Neurol 334:444-465.

Sachse S, Galizia CG (2002) Role of inhibition for temporal and spatial odor representation in olfactory output neurons: a calcium imaging study. J Neurophysiol 87:1106-1117.

Strausfeld NJ (1970) Golgi studies on insects. Part II. The optic lobes of Diptera. Philos Trans R Soc Lond B Biol Sci 258:175-223.

Strausfeld NJ, Blest AD (1970) Golgi study on insects. Part I. The optic lobes of Lepidoptera. Philos Trans R Soc Lond B Biol Sci 258:81-134.

Strausfeld NJ, Nässel DR (1981) Neuroarchitecture of brain regions that subserve the compound eyes of crustacea and insects. In: Handbook of sensory physiology (Autrum H, ed), Vol VII/6B, pp 1-132. New York: Springer.

Strausfeld NJ, Okamura JY (2007) Visual system of calliphorid flies: organization of optic glomeruli and their lobula complex efferents. J Comp Neurol 500:166-188.

Strausfeld NJ, Sinakevitch I, Okamura JY (2007) Organization of local interneurons in optic glomeruli of the dipterous visual system and comparisons with the antennal lobes. Dev Neurobiol 67:1267-1288.

Strauss R (2002) The central complex and the genetic dissection of locomotor behaviour. Curr Opin Neurobiol 12:633-638.

Träger U, Wagner R, Bausenwein B, Homberg U (2008) A novel type of microglomerular synaptic complex in the polarization vision pathway of the locust brain. J Comp Neurol 506:288-300.

Vitzthum H, Muller M, Homberg U (2002) Neurons of the central complex of the locust Schistocerca gregaria are sensitive to polarized light. J Neurosci 22:1114-1125.

Wakakuwa M, Kurasawa M, Giurfa M, Arikawa K (2005) Spectral heterogeneity of honeybee ommatidia. Naturwissenschaften 92:464-467.

Wang Z, Pan Y, Li W, Jiang H, Chatzimanolis L, Chang J, Gong Z, Liu L (2008) Visual pattern memory requires foraging function in the central complex of Drosophila. Learn Mem 15:133-142.

Wehner R (1972) Dorsoventral asymmetry in the visual field of the bee, Apis mellifica. J Comp Physiol 77:256-277.

Wehner R, Rossel S (1985) The bee's celestial compass: a case study in behavioural neurobiology. In: Experimental behavioral ecology and sociobiology (Hölldobler B, Lindauer M, eds), pp 11-54. Stuttgart: Fischer.

Yamaguchi S, Wolf R, Desplan C, Heisenberg M (2008) Motion vision is independent of color in Drosophila. Proc Natl Acad Sci U S A 105:4910 4915 . 